\title{
Influence of sub-surface damage evolution on low-energy-plasma-driven deuterium permeation through tungsten
}

\author{
Stefan Kapser ${ }^{1,2}$, Martin Balden ${ }^{1}$, Tiago Fiorini da Silva ${ }^{1,3}$, \\ Stefan Elgeti ${ }^{1}$, Armin Manhard ${ }^{1}$, Klaus Schmid ${ }^{1}$, \\ Thomas Schwarz-Selinger ${ }^{1}$, Udo von Toussaint ${ }^{1}$ \\ ${ }^{1}$ Max-Planck-Institut für Plasmaphysik, Boltzmannstraße 2, 85748 Garching, Germany \\ ${ }^{2}$ Physik-Department E28, Technische Universität München, James-Franck-Straße 1, \\ 85748 Garching, Germany \\ ${ }^{3}$ Instituto de Física da Universidade São Paulo, Rua do Matão, trav. R 187, \\ 05508-090 São Paulo, Brazil \\ E-Mail: stefan.kapser@ipp.mpg.de, armin.manhard@ipp.mpg.de
}

\begin{abstract}
Low-energy-plasma-driven deuterium permeation through tungsten at $300 \mathrm{~K}$ and $450 \mathrm{~K}$ has been investigated. Microstructural analysis by scanning electron microscopy, assisted by focused ion beam, revealed sub-surface damage evolution only at $300 \mathrm{~K}$. This damage evolution was correlated with a significant evolution of the deuterium amount retained below the plasma-exposed surface. Although both of these phenomena were observed for $300 \mathrm{~K}$ exposure temperature only, the deuterium permeation flux at both exposure temperatures was indistinguishable within the experimental uncertainty. The permeation flux was used to estimate the maximum ratio of solute-deuterium to tungsten atoms during deuterium-plasma exposure at both temperatures and thus in the presence and absence of damage evolution. Diffusion-trapping simulations revealed the proximity of damage evolution to the implantation surface as the reason for an only insignificant decrease of the permeation flux.
\end{abstract}

Keywords: deuterium, plasma, permeation, tungsten, sub-surface damage evolution Submitted to: Nuclear Fusion

\section{Introduction}

Profound knowledge about the interaction of hydrogen isotopes with tungsten is of high relevance for the design of future nuclear fusion reactors, since tungsten is currently 
considered to be very promising for the use as plasma-facing material in such devices (compare, e.g., [1, 2, 3]). For safe operation, retention of hydrogen isotopes (referred to as hydrogen throughout this paper), especially the radioactive tritium, in as well as their permeation through the tungsten need to be minimized [4].

Retention, diffusion deep into the material and permeation can be strongly affected by the presence of material defects (compare, e.g., [4, 5, 6, 7] and references therein). At the same time, the presence of hydrogen in tungsten can also affect the tungsten microstructure. For example, it has been shown that the sub-surface defect structure of tungsten can be modified by deuterium-plasma exposure even far beyond the implantation range [4, 8, 9]. Defect creation by hydrogen and the effect of defects on the hydrogen distribution in tungsten can lead to a complex interplay [6]. This interplay needs to be understood in order to predict hydrogen-isotope retention and recycling in future fusion reactors.

A large number of scientific studies has been performed on the interaction of hydrogenisotope ions with tungsten (see, e.g., [4, 5, 6] and references therein). However, most of these studies were based on measurements of hydrogen retained in the material after ion implantation, which is typically considered to be predominantly trapped at material defects. In contrast to the trapped hydrogen inventory that remains in the tungsten after plasma exposure, the highly mobile interstitially dissolved solute hydrogen typically vanishes afterwards [4] and is thus not measured. However, as the solute is responsible for the diffusive transport of hydrogen [10, 11, it plays a key role in hydrogen retention and permeation. Furthermore, it is also expected to be of crucial importance for defect evolution [4, 12, 13, 14].

The present study reports on measurements of deuterium retention in as well as permeation through tungsten in the presence and absence of sub-surface damage evolution, which represents a modification of the defect structure. The permeation measurement results do not only yield information about the impact of sub-surface damage evolution on the permeation flux, but can also be used to estimate the ratio of solute-deuterium to tungsten atoms present during damage evolution. They thus add crucial information to the incomplete picture derived from retention measurements alone.

\section{Experimental procedure}

\section{$2.1 \quad$ Tungsten samples}

The samples used for deuterium retention and permeation measurements were cut from a cold-rolled tungsten foil purchased from Plansee SE (Austria) with a nominal thickness of $(25 \pm 3) \mu \mathrm{m}$ and a nominal purity of $99.97 \%$ by weight, both values specified by the manufacturer. Before the experiments, all samples were cleaned in an ultrasonic bath in ultra-pure acetone and then in deionized water and finally rinsed in deionized water. Subsequently, all samples were annealed at $2000 \mathrm{~K}$ for $30 \mathrm{~min}$ in vacuum. A number of samples was intentionally cleaved after the experiments to measure their thicknesses on the resulting cross-sections by electron microscopy. These measurements resulted in a mean value for the sample thickness $L$ of about $24.5 \mu \mathrm{m}$, which was used for the plots and simulations presented in this report. The minimum and maximum measured thicknesses were $22 \mu \mathrm{m}$ and $27 \mu \mathrm{m}$, respectively. 


\section{$2.2 \quad$ Deuterium plasma exposure}

To measure plasma-driven deuterium permeation through and retention in the tungsten samples, they were exposed to deuterium plasma in a low-temperature electron cyclotron resonance (ECR) plasma source while keeping the sample holder at floating potential. The plasma source was described in detail in [15]. As mentioned in [16], it has been modified slightly since then, and therefore the deuterium-ion flux to the sample holder has been remeasured with a retarding field analyzer (RFA) (see [15, 16]).

To produce smooth data suited as input for implantation simulations, the differential ion-flux density determined from the RFA signal has been fitted with an analytical function. A sum of three Gaussians and a small constant offset, which is not intended to imply a physical model, was chosen as fit function and resulted in a good fit to the experimental data. Since the RFA measurement yielded the differential ion-flux density with respect to energy, but not the contributions of the individual ion species, the relative abundances of the ion species reported in $[15]^{1}$ of $94 \% \mathrm{D}_{3}^{+}, 3 \% \mathrm{D}_{2}^{+}$and $3 \% \mathrm{D}^{+}$have been used. They are assumed to be unchanged as they depend mainly on the deuterium gas pressure [15], which has not been changed with respect to the standard conditions described in [15]. Furthermore, it has been assumed that all incident ion species have the same energy distribution derived from the RFA measurements, which means the fit function mentioned above, however, with subtracted small offset. This yielded the incident differential deuteron flux density shown in Figure 1. The improved evaluation procedure yielded a total incident deuteron flux density $J_{\text {incident }}$ of $6.0 \times 10^{19} \mathrm{D} / \mathrm{m}^{2} \mathrm{~s}$, which represents only a minor correction of five percent compared to the value in [16], where the same experimental data was used.

All samples were pre-sputtered in-situ by argon plasma with a sample-holder bias of $-100 \mathrm{~V}$ before deuterium-plasma exposure to gain clean and reproducible surface conditions. To determine the sample temperature during plasma exposure, the sample holder temperature was measured with a thermocouple pressed against its backside as described in [15]. As the samples were firmly clamped to the sample holder, it appears justified to assume that the sample temperature was at all times very close to the sample-holder temperature, which is subsequently given as the exposure temperature. The sample holder was cooled or heated by thermostats operating with ethanol or oil.

All deuterium-plasma exposures were carried out without interruption, except for the $336 \mathrm{~h}$ exposures. These were obtained by exposing the $192 \mathrm{~h}$ exposed samples for another $144 \mathrm{~h}$ after a first analysis.

\subsection{NRA retention and permeation measurements}

The deuterium retention in the tungsten samples after plasma exposure was studied by nuclear reaction analysis (NRA) using the nuclear reaction $\mathrm{D}\left({ }^{3} \mathrm{He}, \mathrm{p}\right)^{4} \mathrm{He}$ [18, 19] with ${ }^{3} \mathrm{He}$ under normal incidence and two proton detectors with solid angles of $30.3 \mathrm{msr}$ and $77.5 \mathrm{msr}$, both under a reaction angle of $135^{\circ}$.

The retained deuterium depth profiles in tungsten after plasma exposure were determined from the proton spectra using NRADC [20]. As described in detail in [20, NRADC is a computer program for the determination of depth profiles of trace impurities from ionbeam-analysis (IBA) data. The most probable depth profile is determined by matching a

\footnotetext{
${ }^{1}$ With respect to the relative abundances of the ion species given in [15], please also note the remark to [15] included in the reference list of [17.
} 


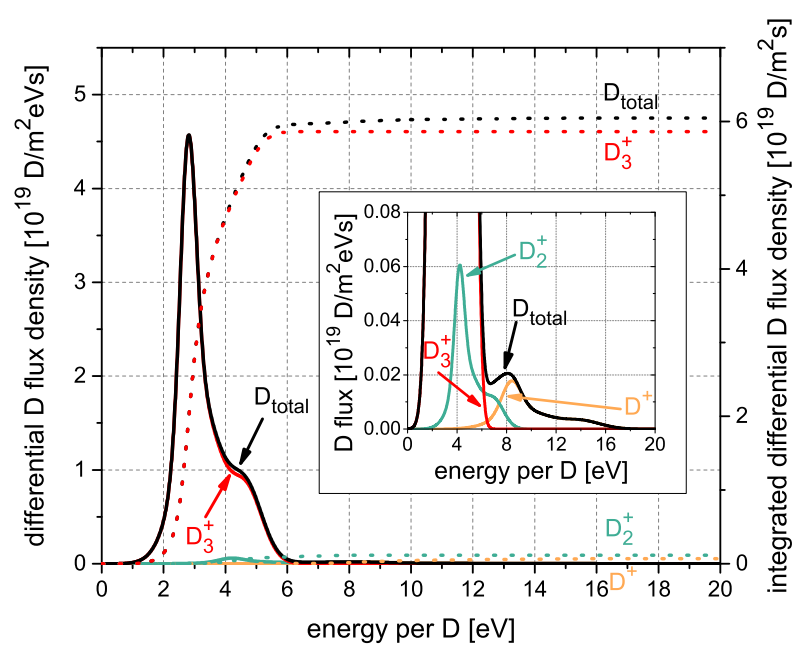

Figure 1: Total incident differential deuteron flux density and contributions by different deuterium ion species (solid lines) as well as their cumulative integrals (dashed lines). The spectrum is based on measurements of the differential ion-flux density with a retarding field analyzer, which were already mentioned in [16], and the relative abundances of the deuterium ion species reported in [15. It is dominated by $\mathrm{D}_{3}^{+}$ions, with minor contributions of $\mathrm{D}_{2}^{+}$and $\mathrm{D}^{+}$. The low incident energy avoids kinetic defect creation in tungsten exposed to the plasma. The insert shows a magnified view of the contributions by $\mathrm{D}^{+}$and $\mathrm{D}_{2}^{+}$.

forward calculation of IBA spectra to the experimental data using a maximum-likelihood approach. To improve the computational efficiency, NRADC does not perform a full forward calculation of the physical model in each analysis step, but linearizes the problem based on a single set of forward calculations generated using the computer program SIMNRA [21, 22]. Since SIMNRA assumes element depth profiles consisting of layers with constant composition, the forward calculations are performed on a finely resolved layer structure. These so-called sub-layers are then binned into larger layers by NRADC. This procedure improves the speed of the calculations, but limits the analysis method to low concentrations of the investigated trace element that do not contribute significantly to the stopping power. This is fulfilled for deuterium retained in tungsten at the atomic fractions presented in this report (compare [20]). The description by layers of constant composition is of course an approximation of the underlying, typically continuous, concentration profiles.

An uncertainty estimate for the resulting depth profile is a challenging task, because it needs to take the uncertainty in the trace-element atomic fraction of each layer as well as the uncertainty in the layer thicknesses into account. Since the atomic-fraction probability distribution in each layer can be complex, e.g. bimodal, a representation by error bars or confidence intervals can almost always only be a simplified representation. A plot of the full histogram of the resulting probability distribution of the estimated atomic fraction within one layer is in principle possible and would contain the full information, but would be confusing when comparing different depth profiles in a single graph. Thus, the deuterium depth profiles presented in this report include the most probable depth profile, accompanied by an uncertainty band with the upper and lower bound given by exclusion of the highest and lowest $2.5 \%$ of the probability distribution in each sub-layer, which thus encompasses $95 \%$ of the probability distribution. While the discretization of the most probable depth profile is determined by the most probable layer number, the 
discretization of the uncertainty band limits is based on the higher resolved sub-layer structure.

While there is a formal difference between the deuterium atomic fraction in tungsten and the ratio of deuterium to tungsten atoms, this difference is negligible for the atomic fractions given in this report. Therefore, the two terms are used synonymously here.

Before deuterium-plasma exposure, samples for permeation measurements were coated with a layer system on the side that was not intended to be exposed to deuterium plasma. This side will subsequently be referred to as the permeation side. The procedure used for the permeation measurements, which is sketched schematically in Figure 2, has been described in detail in [16] and will only briefly be repeated here, including a few improvements.

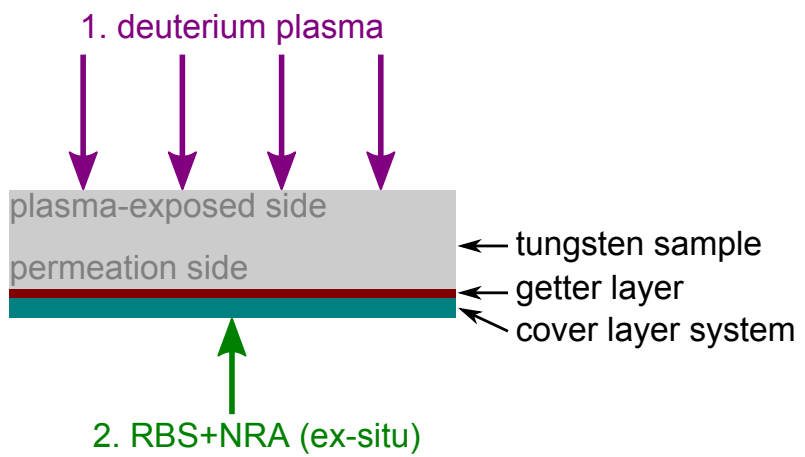

Figure 2: Procedure used for the permeation measurements, as described in detail in [16]. A getter layer on the permeation side of the tungsten samples is used to accumulate permeated deuterium during plasma exposure. Subsequently, the amount of permeated deuterium is determined using a combination of ex-situ Rutherford backscattering spectroscopy (RBS) and nuclear reaction analysis (NRA). A cover layer system prevents direct uptake of deuterium from the background deuterium gas during plasma exposure and helps to distinguish deuterium in the getter and at the cover surface. Relative layer thicknesses are not drawn to scale.

A getter layer of either zirconium (Zr), titanium (Ti) or erbium (Er) with a thickness of about $300 \mathrm{~nm}$ on the permeation side of the sample is used to accumulate the permeated deuterium. A cover layer system, which consists of layers of tungsten $(\approx 50-75 \mathrm{~nm})$, copper $(\approx 950 \mathrm{~nm})$, tungsten $(\approx 50-75 \mathrm{~nm})$ and erbium oxide $(\approx 400 \mathrm{~nm})$ and is deposited on top of the getter, is used to prevent direct loading of the getter from the deuterium background gas pressure during plasma exposure. In addition, it helps to distinguish deuterium in the getter and at the cover surface. As also already described in [16], a number of samples has been exposed to the background deuterium gas, but masked on the plasma-exposed side to prevent implantation of any incident deuterium ions. This was done to test the impermeability of the cover layer system. As a negligible deuterium amount in the getter of masked samples (compare [16] and Figure 9p demonstrates, the deuterium amount in the getter is not significantly increased by permeation through the cover. Thus, it can be concluded that the resulting deuterium amount retained in the getter after plasma exposure is essentially equal to the time integral of the permeation flux during plasma exposure. The additional amount contributed by out-diffusion of deuterium present in the tungsten at the end of the plasma exposure into the getter is negligible according to our diffusion-trapping simulations (for simulation parameters see Section 4).

As described in [16], sputter x-ray photo-electron spectroscopy (XPS) measurements 
suggested some oxygen at the interface between tungsten foil and getter layer. Therefore, the argon pre-sputtering procedure before the getter layer deposition has been improved by increasing the injected power used for substrate cleaning by sputtering. Most, but not all, of the permeation data for plasma exposure at $300 \mathrm{~K}$ presented in this report has been generated from measurements already described in [16. The samples used for these measurements were produced with the old pre-sputtering procedure. In contrast, most permeation data for plasma exposure at $450 \mathrm{~K}$ has been produced from samples deposited with the new pre-sputtering procedure. However, no inconsistencies between permeation-measurement results from samples of both batches have been observed. For example, permeation data for $120 \mathrm{~h}$ deuterium-plasma exposure at $300 \mathrm{~K}$ that originates from measurements on samples produced with the new pre-sputtering procedure (and has been obtained independently from the $120 \mathrm{~h}$ data presented in [16]) is consistent with the rest of the permeation data for exposure at $300 \mathrm{~K}$ within the observed data scatter (compare also Figure 9p. This shows that the differences between the old and new pre-sputtering procedure before getter layer deposition have no significant effect on the measured permeated deuterium amount.

Layer structure and composition, including the deuterium amount in the getter, are determined using a combination of Rutherford backscattering spectroscopy (RBS) data from one location on each sample and nuclear reaction analysis (NRA) data from multiple locations on each sample. The RBS spectra are analyzed to determine the structure and composition of the layer system of each sample. This information is then used in SIMNRA [21, 22] simulations of the nuclear reaction between incident ${ }^{3} \mathrm{He}$ and deuterium in the sample. Based on the simulation results, the deuterium amount in the getter is determined from NRA proton spectra by matching the simulated peak integrals to the experimental ones. For the NRA of deuterium accumulated in the getter, the same reaction and proton detectors as described above for the retention measurements were used. The additional inclusion of spectra from the second proton detector in this analysis improved the counting statistics compared to [16].

The determination of the layer structure and composition has been improved compared to [16] by fitting the RBS signal from all layers simultaneously using MultiSIMNRA [23], which uses SIMNRA [21, 22 for physics calculations. The objective function used was the regular $\chi^{2}$ instead of the reduced $\chi^{2}$, which is mentioned in [23]. Furthermore, a small amount of hafnium present in the zirconium getter that resulted from a small amount of hafnium in the zirconium sputter target has now been taken into account.

While in [16] roughness was taken into consideration in the RBS fits directly using SIMNRA, it was omitted in the present evaluation, because it would have dramatically increased the computation time in combination with the simultaneous fitting of multiple parameters. Such long computation times appeared unfeasible for the large number of measurements. The error introduced in the determination of the deuterium amount in the getter by this approximation was estimated by fitting a few datasets in MultiSIMNRA with roughness enabled for the substrate and the top cover layer. Comparison of the final results including and excluding roughness yielded a difference in the determined deuterium amount in the getter of around two percent, which appears negligible compared to the data scatter.

Estimating the uncertainties in the permeated deuterium amount is challenging due to the large number of parameters involved. These include uncertainties in, e.g., stopping power and scattering cross-sections of the various elements, roughness of substrate and layers as well as counting statistics. As mentioned in [16], the main systematic uncertainty 
is assumed to be caused by the assumption of a homogeneous deuterium distribution over the getter thickness, which may lead to a maximum overestimate of about ten percent. With respect to the total statistical uncertainty, the data scatter of measurements on the same sample is assumed to be a good indication.

As the deuterium-containing getter layer interferes with investigation of deuterium retention in the tungsten foil below the permeation side, samples without layer system were used for this purpose.

\subsection{Thermal desorption spectroscopy}

In addition to NRA measurements on plasma-exposed and permeation side, samples without layer system were also investigated by thermal desorption spectroscopy (TDS).

TDS was performed in the glass tube of the TESS setup, of which a detailed description can be found in [24]. In this setup, the sample under investigation is located in an evacuated quartz-glass tube and heated by a tube furnace. The desorbing species can be detected and distinguished using a quadrupole mass spectrometer (QMS). For the present study, the furnace was heated with a ramp of $15 \mathrm{~K} / \mathrm{min}$ from room temperature to a maximum temperature of about 1300 K. Simultaneously, the desorption of selected species was monitored with the QMS. All desorption fluxes in this report are normalized to the plasma-exposed area on the samples, which was about $(10 \times 10) \mathrm{mm}^{2}$.

Calibration of the sample temperature was performed by reheating some already measured samples with the same furnace temperature ramp, this time with a thermocouple spot-welded to them. The $\mathrm{D}_{2}$ signal was calibrated with a calibrated leak. The HD calibration factor was calculated based on the measured $\mathrm{D}_{2}$ calibration factor, using the ratio of the $\mathrm{HD}$ and $\mathrm{D}_{2}$ calibration factors given in 25. . Heavy water species are difficult to use for a quantitative as well as qualitative analysis because of their large sticking coefficient to surfaces, e.g. chamber walls, between sample and quadrupole mass spectrometer [25]. Keeping the associated uncertainties in mind, the deuterium amount carried by heavy water was estimated using calibration factors calculated based on the measured $\mathrm{D}_{2}$ calibration factor and the relative sensitivity factors for deuterium and water reported in [26].

\subsection{Microstructural analysis}

Microstructural investigations were carried out using a Helios NanoLab 600 dual beam setup manufactured by FEI. It consists of a scanning electron microscope (SEM) and a focused ion beam (FIB) device.

The SEM images presented in this report were recorded either with an EverhardThornley detector (ETD) or a segmented concentric backscatter (CBS) detector. Images with different contrast were recorded using different segments of the CBS detector, which was subdivided into an inner ring and a three-part outer ring.

Two modes were used to record the CBS images included in this report. Images intended to visualize surface topography were generated by the difference of the signals from two outer-ring parts at an electron acceleration voltage of $5 \mathrm{kV}$ and are referred to as topographic-contrast images. Images intended to visualize crystal distortion were recorded using the sum of all segments of the CBS detector with an electron acceleration voltage of $30 \mathrm{kV}$ and are here referred to as orientation-contrast images. When used to visualize material contrast on samples consisting of different materials, such images are 
typically referred to as Z-contrast images. The presented ETD images were recorded in secondary electron (SE) mode using an acceleration voltage of $5 \mathrm{kV}$.

\section{Experimental results}

\subsection{Sub-surface damage evolution}

Figures $3 \mathrm{a}$ )-c) show orientation-contrast SEM images of plasma-exposed tungsten surfaces after exposure to deuterium plasma for $24 \mathrm{~h}, 96 \mathrm{~h}$ and $144 \mathrm{~h}$ at $300 \mathrm{~K}$. This corresponds to fluences of about $5.2 \times 10^{24} \mathrm{D} / \mathrm{m}^{2}, 2.1 \times 10^{25} \mathrm{D} / \mathrm{m}^{2}$ and $3.1 \times 10^{25} \mathrm{D} / \mathrm{m}^{2}$, respectively. In comparison with an unexposed reference sample, as displayed in Figure $3 \mathrm{~d}$ ) with the same contrast mode, in-grain damage features are clearly visible after deuterium-plasma exposure for $96 \mathrm{~h}$ and $144 \mathrm{~h}$. The analysis is complicated by the fact that the feature visibility was strongly dependent on grain orientation and observation direction, which lets a full quantitative analysis seem unreliable. The displayed regions of the sample surfaces were selected attempting to give an impression of the average value and variation of the damage-feature areal density observed in the region inspected by SEM. Based on Figures 3 a)-c), the number of damage features appears to increase with exposure time. However, the surface of a sample inspected after $192 \mathrm{~h}$ of deuterium-plasma exposure at $300 \mathrm{~K}$ deviated from this trend: It appeared to have a lower areal density of damage features than Figure 3c) and even slightly lower than Figure 3b). The observed evolution of damage features due to the deuterium-plasma exposure at $300 \mathrm{~K}$ thus had a significant data scatter. This will also be important for the discussion of the scatter in the deuteriumretention data in Section 3.2 .

While a significant number of damage features is visible in the orientation-contrast image recorded after $144 \mathrm{~h}$ deuterium-plasma exposure at $300 \mathrm{~K}$ displayed in Figure 3c), no significant damage-feature evolution was observed for the same plasma-exposure conditions at $450 \mathrm{~K}$ even after $336 \mathrm{~h}$ of deuterium plasma exposure (carried out in two steps of $192 \mathrm{~h}$ and $144 \mathrm{~h}$ ), as displayed in Figure 3e). This plasma-exposure duration corresponds to a fluence of about $7.3 \times 10^{25} \mathrm{D} / \mathrm{m}^{2}$.

A comparison of Figure 3c) with an image of the same surface region recorded in topographic contrast mode, displayed in Figure 3f), reveals no visible surface elevation at the locations of the damage features. It thus does not appear to be justified to refer to these features as "blisters".

Figure 4 shows a topview orientation-contrast image including multiple damage features observed on a tungsten surface exposed to deuterium plasma for $144 \mathrm{~h}$ at $300 \mathrm{~K}$ together with an image of a cross-section prepared by FIB at the location of several damage features and imaged by SEM using the ETD. The cross-section reveals a number of sub-surface material defects in depths of up to about one micron below the surface. Their positions are in agreement with the positions of damage features visible in the corresponding topview orientation-contrast image. Since the exact nature of the sub-surface defects is not known, they are subsequently referred to as sub-surface damage.

In sum, the microscopic analysis revealed sub-surface damage evolution by deuteriumplasma exposure, which was only observed for an exposure temperature of $300 \mathrm{~K}$, but not for $450 \mathrm{~K}$, for the given experimental conditions. The dependence of the areal density of damage features on the deuterium-plasma exposure time showed indications for a continuous increase, but could not be fully clarified. This was due to significant data scatter, which was partially caused by the dependence of feature visibility on grain orientation 


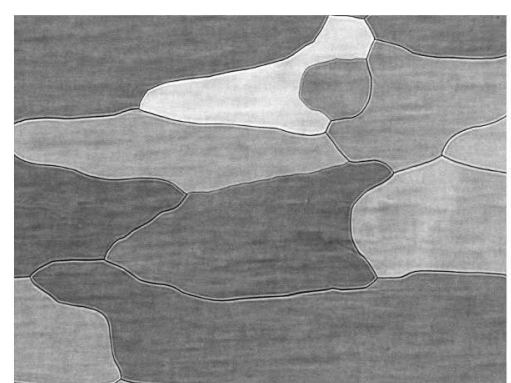

a) $300 \mathrm{~K}, 24 \mathrm{~h}$

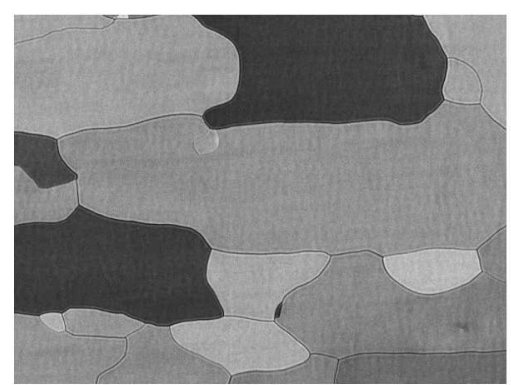

d) unexposed reference

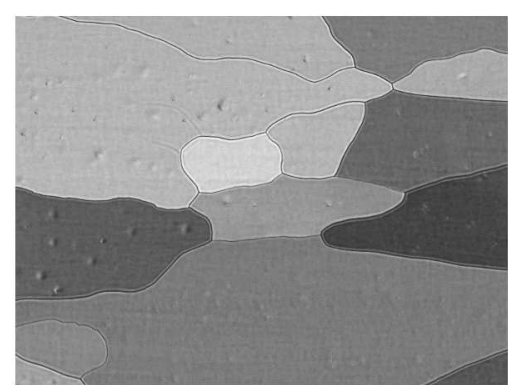

b) $300 \mathrm{~K}, 96 \mathrm{~h}$

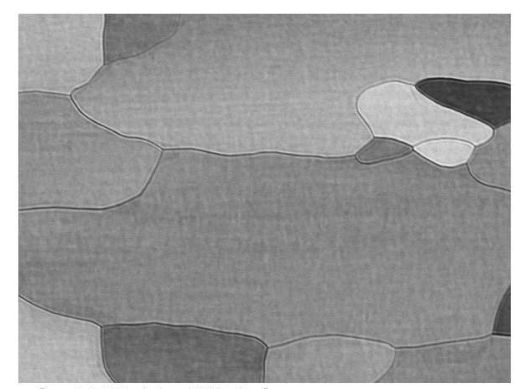

e) $450 \mathrm{~K}, 336 \mathrm{~h}$

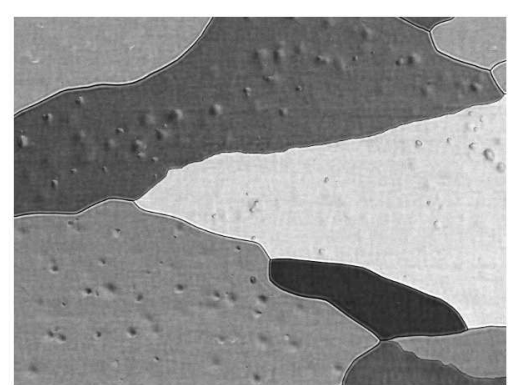

c) $300 \mathrm{~K}, 144 \mathrm{~h}$

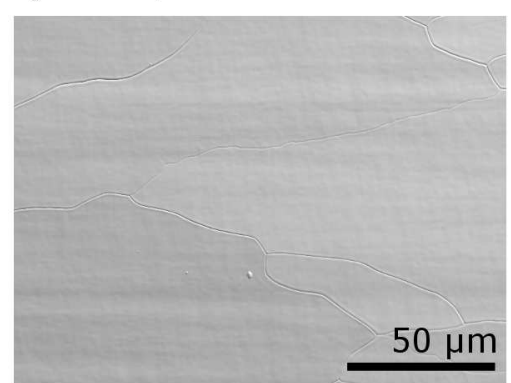

f) $300 \mathrm{~K}, 144 \mathrm{~h}$, topography

Figure 3: Damage features observed on deuterium-plasma-exposed tungsten surfaces by SEM. Orientation-contrast images a)-c), recorded with all segments of a concentric backscatter detector, show the surface after deuterium-plasma exposure at $300 \mathrm{~K}$ for $24 \mathrm{~h}$, $96 \mathrm{~h}$ and $144 \mathrm{~h}$, respectively. While damage-feature evolution at $300 \mathrm{~K}$ is clearly visible, no significant damage-feature evolution was observed for deuterium-plasma exposure at $450 \mathrm{~K}$ even for $336 \mathrm{~h}$ exposure time, as displayed in e), where the same contrast mode was used. Images generated by subtracting the signals from two parts of the backscatter detector, yielding a topographic-contrast image, showed no indication for surface elevation at the damage-feature locations as a comparison of the topographic-contrast image f) with an orientation-contrast image of the same region c) shows. An orientation-contrast image of an unexposed tungsten surface is included as a reference in d). The scale bar in $\mathrm{f})$ is valid for all images. 


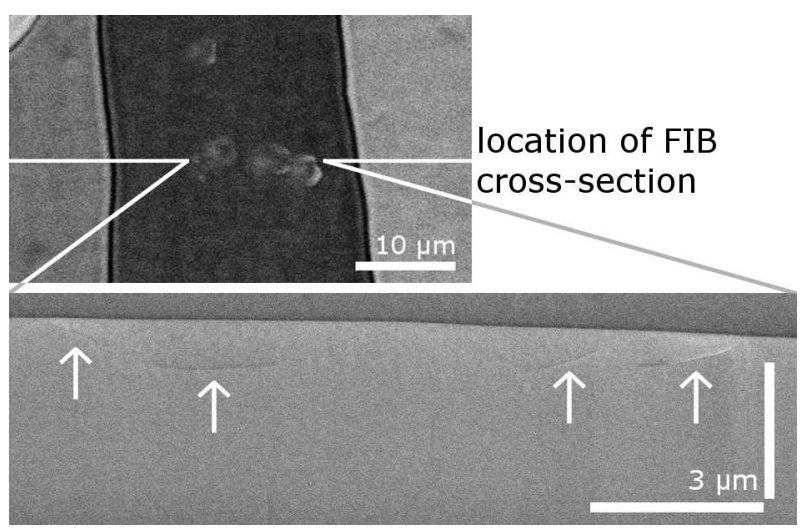

Figure 4: Topview orientation-contrast SEM image of a number of damage features observed on a tungsten surface exposed to deuterium plasma at $300 \mathrm{~K}$ for $144 \mathrm{~h}$ (upper image) and ETD SEM image of a FIB-prepared cross-section at the location of several of these damage features (lower image). For the latter, the viewing direction was $38^{\circ}$ with respect to the surface normal of the cross-section. The FIB cross-section reveals sub-surface damage (marked with arrows) below damage features observed in the topview orientation-contrast image.

and observation direction, but probably mainly by other factors such as small differences in the initial microstructure.

\subsection{Deuterium retention}

To study the deuterium retention in the tungsten samples below plasma-exposed and permeation side after low-energy-deuterium-plasma exposure, samples without layer system were exposed to deuterium plasma for $12 \mathrm{~h}$ and $192 \mathrm{~h}$, corresponding to fluences of about $2.6 \times 10^{24} \mathrm{D} / \mathrm{m}^{2}$ and $4.2 \times 10^{25} \mathrm{D} / \mathrm{m}^{2}$, respectively, both at $300 \mathrm{~K}$ and $450 \mathrm{~K}$. They were subsequently analyzed by NRA performed on the plasma-exposed as well as the permeation side and the data was analyzed using NRADC [20]. The resulting deuterium depth profiles are displayed in Figure 5.

All these depth profiles show a surface retention peak in the first layer at plasmaexposed and permeation side, which is commonly attributed to a surface adsorbate. This is in contrast to deuterium in deeper layers, which was retained in the tungsten bulk. Please note that the displayed surface-layer thickness of about $150 \mathrm{~nm}$ is determined by the measurement resolution, while the actual thickness of the adsorbate is expected to have been smaller. Such surface retention peaks have therefore been neglected in the subsequent analysis and discussion regarding these and other depth profiles. This appears well justified, also because the total amount of deuterium contained in each surface layer was at maximum about $3 \times 10^{18} \mathrm{D} / \mathrm{m}^{2}$, which is significantly less than a monolayer.

For $12 \mathrm{~h}$ exposure time, the deuterium depth profiles in the NRA range of about $8 \mu \mathrm{m}$ below the plasma-exposed surface for both exposure temperatures are very similar. In both cases, a deuterium atomic fraction of the order of $10^{-5}$ was present below the surface, which slightly decreased deeper into the bulk. The amount of deuterium retained below the permeation-side surface for this exposure time was negligible.

In contrast to the $12 \mathrm{~h}$ data, the deuterium depth profiles below the plasma-exposed surface for $192 \mathrm{~h}$ of deuterium-plasma exposure are significantly different for the two exposure temperatures. The deuterium atomic fraction after $192 \mathrm{~h}$ exposure at $450 \mathrm{~K}$ 


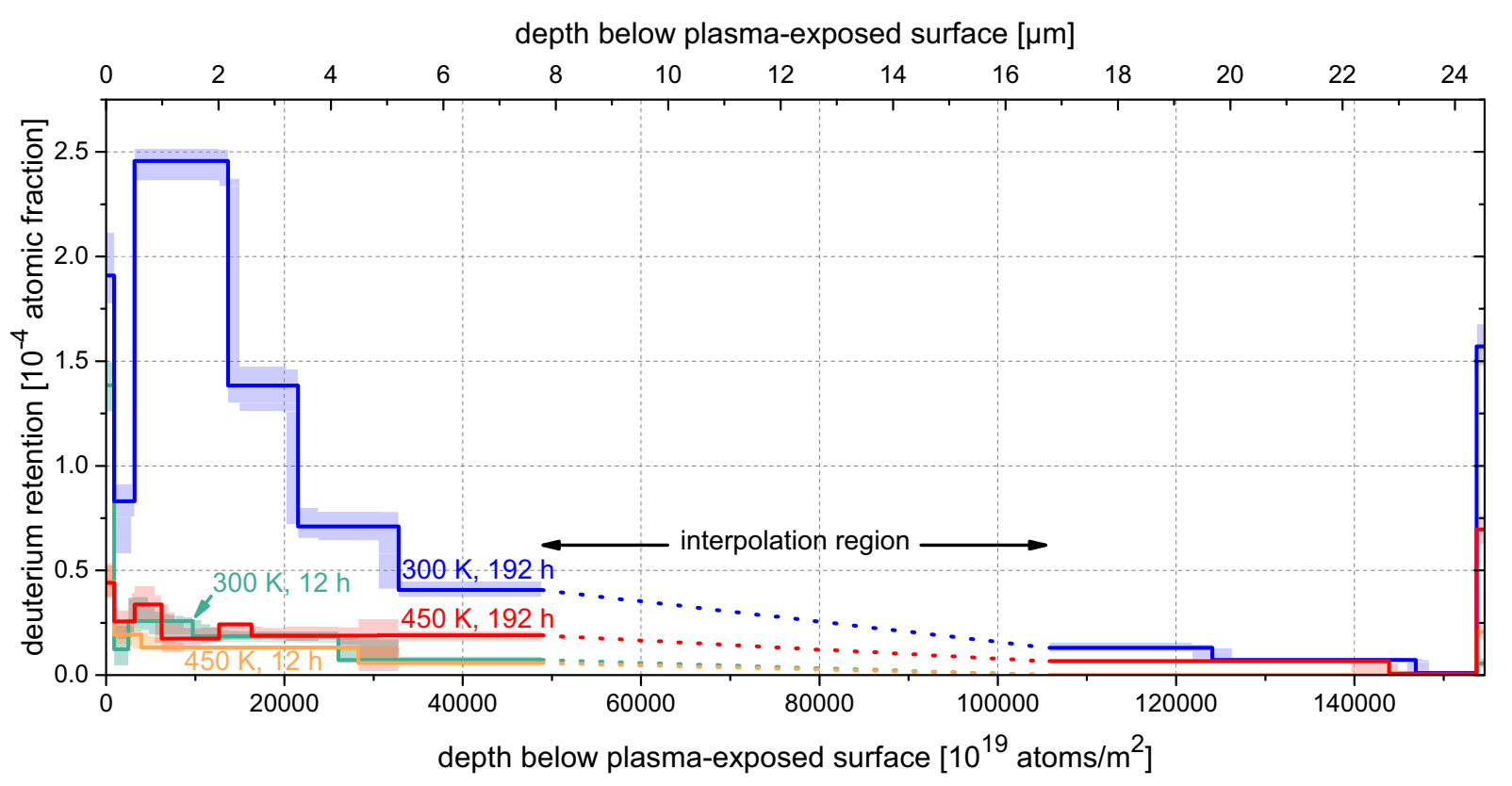

Figure 5: Depth profiles of deuterium retained in tungsten after deuterium-plasma exposure at $300 \mathrm{~K}$ and $450 \mathrm{~K}$ determined by NRA on the plasma-exposed and permeation side. The exposure times of $12 \mathrm{~h}$ and $192 \mathrm{~h}$ correspond to incident fluences of about $2.6 \times 10^{24} \mathrm{D} / \mathrm{m}^{2}$ and $4.2 \times 10^{25} \mathrm{D} / \mathrm{m}^{2}$, respectively. The middle of the sample, which lies outside the NRA ranges, was interpolated linearly with dotted lines to guide the eye.

was comparable to the $12 \mathrm{~h}$ value, but for $300 \mathrm{~K}$ exposure temperature it was much higher. However, in the NRA range below the permeation side, the deuterium atomic fractions after $192 \mathrm{~h}$ for both exposure temperatures were comparable.

The deuterium atomic fraction retained within several $\mu \mathrm{m}$ below the plasma-exposed surface after $12 \mathrm{~h}$ and $192 \mathrm{~h}$ exposure at $450 \mathrm{~K}$ shows no significant difference. In contrast, the deuterium atomic fraction was about a factor of ten different for the same exposure times with an exposure temperature of $300 \mathrm{~K}$. Therefore, the development of the deuterium retention in the bulk below the plasma-exposed surface with exposure time for both exposure temperatures is now discussed in detail, also to elucidate a possible correlation with the observation of sub-surface damage evolution described above.

The bulk deuterium amount, excluding the surface layer, in the NRA range below the plasma-exposed surface is plotted in Figure 6 over the deuterium-plasma exposure time. Data from samples without layer system used to generate Figure 5 is included as well as data determined from NRA measurements on the plasma-exposed side of samples with a layer system on the permeation side. For a number of selected samples, detailed deuterium depth profiles were determined based on NRA measurements with eight incident ${ }^{3} \mathrm{He}$ energies in the range from $500 \mathrm{keV}$ to $4500 \mathrm{keV}$ using NRADC. Because of the low deuterium content, total charges of either $20 \mu \mathrm{C}$ or $50 \mu \mathrm{C}$ were accumulated for each energy with a beam spot of approximately $1 \mathrm{~mm}^{2}$ to gain sufficient counting statistics. This led to very long measurement times especially at low incident ${ }^{3} \mathrm{He}$ energies, which resulted in measurement times in the hour-range per depth profile.

To improve on the number of samples that could be analyzed, additional samples were investigated with a reduced set of selected ${ }^{3} \mathrm{He}$ energies. As the basic shape of the profile changes only little with exposure time (compare Figure 7), measurements with only two incident ${ }^{3} \mathrm{He}$ energies appeared sufficient to estimate the total deuterium amount in 
the NRA range. The proton-peak integrals for incident ${ }^{3} \mathrm{He}$ energies of $1800 \mathrm{keV}$ and $4500 \mathrm{keV}$ of the depth profiled samples were used to determine proportionality factors between the proton peak integral and the total deuterium amount in the NRA range in these cases. For these samples, mean values of the calibration factors were calculated for each combination of the incident energies $1800 \mathrm{keV}$ and $4500 \mathrm{keV}$ and the exposure temperatures $300 \mathrm{~K}$ and $450 \mathrm{~K}$. Subsequently, these mean calibration factors were used to determine the total deuterium amount in the NRA range below the plasma-exposed surface based on the proton peak integrals from measurements at $1800 \mathrm{keV}$ and $4500 \mathrm{keV}$ incident ${ }^{3} \mathrm{He}$ energy for those samples where a full depth profile was available as well as for those that were investigated only with two incident ${ }^{3} \mathrm{He}$ energies. Finally, the mean value of the total deuterium amounts in the NRA range determined based on the $1800 \mathrm{keV}$ and $4500 \mathrm{keV}$ proton peak integrals was calculated to determine a final estimate of the total deuterium amount in the NRA range, thus additionally compensating the effect of small variations in the profile shape. As is shown in Figure 6, the deuterium amounts determined based on proton peak integrals from samples where full depth profiles were available are in excellent agreement with the deuterium amounts determined from the full depth profiles. Thus, also the deuterium amounts determined with the same method for samples that were investigated with only two incident ${ }^{3} \mathrm{He}$ energies are assumed to be reliable.

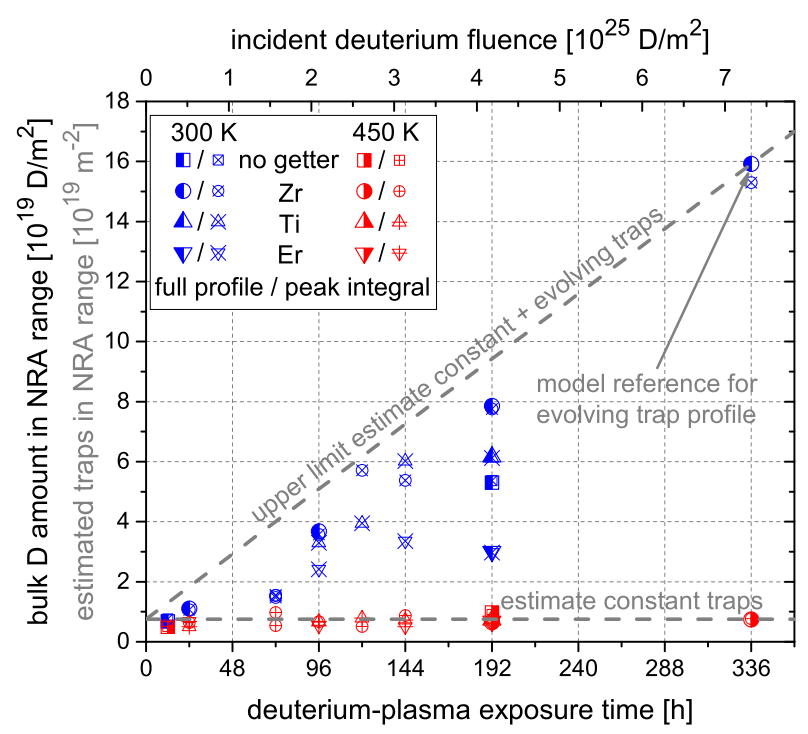

Figure 6: Time evolution of the bulk D amount in the NRA range of about $8 \mu \mathrm{m}$ below the plasma-exposed surface for $300 \mathrm{~K}$ and $450 \mathrm{~K}$ exposure temperature. Half-filled symbols are based on full deuterium depth profiles determined with NRADC [20] from NRA measurements at eight different incident ${ }^{3} \mathrm{He}$ energies. Open symbols with crosses were determined from measurements at two incident ${ }^{3} \mathrm{He}$ energies using mean proportionality factors for the ratio of proton peak integrals and deuterium amounts determined based on the NRADC depth profiles (see text for details). The data for samples without getter layer at $12 \mathrm{~h}$ and $192 \mathrm{~h}$ originates from the same measurements as the depth profiles displayed in Figure 5.

Figure 6 shows that for deuterium-plasma exposure at $450 \mathrm{~K}$, the retained bulk deuterium amount below the plasma-exposed surface stays constant with increasing deuteriumplasma exposure time, while for an exposure temperature of $300 \mathrm{~K}$ most data points indicate an increase of the retained deuterium amount with exposure time, albeit with a 
significant data scatter. The constant retained amount at the level of the data for $450 \mathrm{~K}$ also represents a natural choice as a lower-limit estimate for deuterium retention after exposure at $300 \mathrm{~K}$. This case could be well described by a constant background trap density, also commonly referred to as intrinsic trap density, which is being filled to an equilibrium value and then contains a constant deuterium concentration. The average of the $450 \mathrm{~K}$ bulk deuterium amounts from NRADC depth profiles for exposure times of $192 \mathrm{~h}$ and above was used to estimate the average trapped deuterium amount in these background traps in the NRA range below the plasma-exposed surface.

Under the assumption that each trap can retain zero or one deuterium atoms and all traps in the NRA range below the plasma-exposed surface are completely filled for the given experimental conditions, this value would be identical to the number of background traps in the NRA range. The assumption that all these traps are completely filled appears at least approximately justified, e.g., because Figure 5 shows very similar retention for both temperatures after $12 \mathrm{~h}$ exposure, where trap generation appears to not yet dominate retention at $300 \mathrm{~K}$.

The thus estimated amount of intrinsic background traps in the NRA range is indicated by the lower dashed gray line in Figure 6. From this value, an average number of background traps per tungsten atom of $\rho_{0}^{\mathrm{tr}}=1.5 \times 10^{-5}$ was calculated, which is assumed to be present in the whole sample. It is also in good agreement with the measured deuterium depth profiles in Figure 5.

The exact type of the background traps is presently not known. Possible candidates are dislocations and grain boundaries [27], but also impurity atoms and possibly vacancies [28].

Within the framework of these assumptions, the increased retention observed for $300 \mathrm{~K}$ exposure temperature is assumed to result from an increased trap density, and hence trapped deuterium amount, within the NRA range below the plasma-exposed surface.

An upper limit of trap generation at $300 \mathrm{~K}$ was estimated by assuming a linear increase of the retained deuterium amount in the NRA range, starting at time zero from the estimated amount in background traps and going through the data point recorded for a plasma exposure at $300 \mathrm{~K}$ for $336 \mathrm{~h}$ that is based on a full NRADC depth profile. This appears to be a reasonable upper-limit estimate, as all other bulk deuterium amounts displayed in Figure 6 are below this limit. It leads to an upper-limit estimate for the rate with which deuterium gets trapped in the evolving traps in the NRA range below the plasma-exposed side of about $R_{\mathrm{NRA}}^{\mathrm{D}}=1.3 \times 10^{14} \mathrm{D} / \mathrm{m}^{2} \mathrm{~s}$. Keeping the assumption already made above that each trap can retain zero or one deuterium atoms and all traps in the NRA range below the plasma-exposed surface are completely filled, this yields an upperlimit estimate for the trap generation rate in the NRA range below the plasma-exposed surface of about $R_{\mathrm{NRA}}^{\mathrm{tr}}=1.3 \times 10^{14} \mathrm{~m}^{-2} \mathrm{~s}^{-1}$. Up to a deuterium fluence of $7.3 \times 10^{25} \mathrm{D} / \mathrm{m}^{2} \mathrm{~s}$, which is the maximum fluence that was investigated here, no indications for saturation were found.

Besides the time evolution of the total deuterium amount in the NRA range below the plasma-exposed surface, also the time evolution of the profile shape of the retained deuterium is of interest. The deuterium depth profiles determined for samples with a $\mathrm{Zr}$ getter exposed at $300 \mathrm{~K}$ are displayed in Figure 7. They give a good representation of the evolving deuterium retention profile close to the above-mentioned upper-limit estimate because their corresponding data points in Figure6 are close to the line that indicates the upper-limit estimate. A peaked deuterium profile with a maximum in a depth of about $1.5 \mathrm{\mu m}$ evolves. The estimated number of background traps per tungsten atom $\rho_{0}^{\operatorname{tr}}$, which 
is in good agreement with the experimental data, is also indicated in Figure 7 as a dashed gray line.

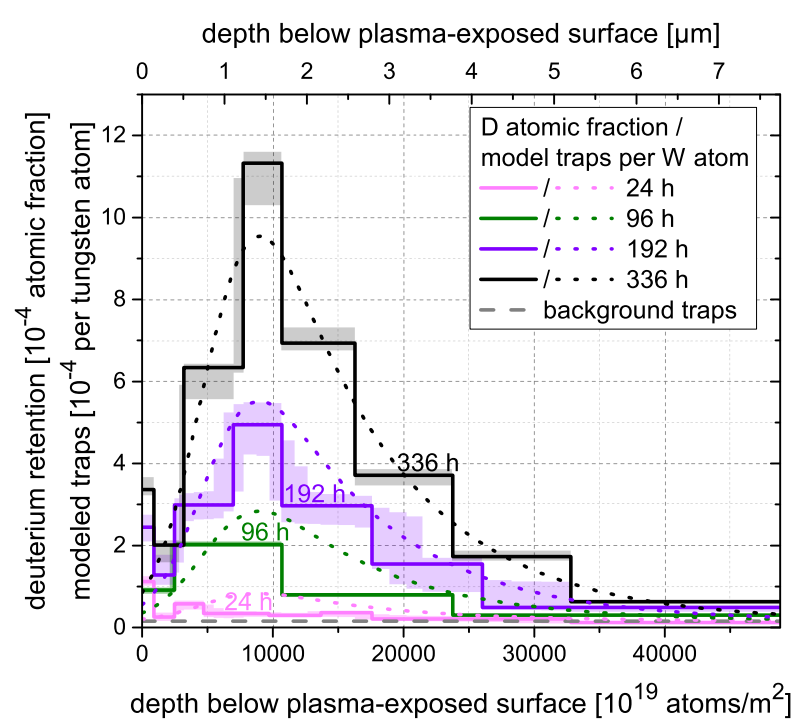

Figure 7: Deuterium depth profiles below the plasma-exposed surface (solid lines) of samples with a Zr getter exposed to deuterium plasma at $300 \mathrm{~K}$ (compare also Figure 6) for different exposure times. A smooth time-dependent function used for modeling of the corresponding traps per tungsten atom is also included (dotted lines). Furthermore, the assumed constant number of background traps per tungsten atom is indicated (dashed gray line).

Combining the damage-feature evolution discussed in Section 3.1 with the retention data in Figure 6, similarities between sub-surface damage evolution and deuterium retention can be found. Therefore, it is important to know that the images displayed in Figures 3 a)-c), which indicate a continuous increase of the damage-feature areal density with exposure time, originate from the same samples as the $\mathrm{Zr}$ data points with the corresponding exposure times in Figure 6. It is striking that all these data points are close to the upper-limit estimate and represent a continuous increase also of the deuterium retention below the plasma-exposed side with exposure time. In contrast, the sample exposed to deuterium plasma for $192 \mathrm{~h}$ at $300 \mathrm{~K}$ mentioned in Section 3.1 that showed even less damage features than the sample of Figure $3 \mathrm{~b}$ ) after $96 \mathrm{~h}$ exposure time, corresponds to the $192 \mathrm{~h}$ Er getter data point in Figure 6. As with the areal density of damage features, also the deuterium retention below the plasma-exposed side of this sample is thus lower than for the samples corresponding to Figures $3 \mathrm{~b}$ ) and c). This strongly indicates a correlation of sub-surface damage evolution and deuterium retention.

Since no correlation between the scatter in sub-surface deuterium retention and the permeated deuterium amount determined with different getter materials (compare Figure 9) was observed, the different getter materials can be excluded as the origin of the data scatter in damage evolution and deuterium retention. Rather, other uncertainties such as variations in the initial microstructure appear likely to be responsible for the observed scatter in damage evolution and deuterium retention below the plasma-exposed surface.

The observed correlation of evolving sub-surface damage and increased deuterium retention at $300 \mathrm{~K}$ as well as absence of both at $450 \mathrm{~K}$ strongly suggests increased retention of deuterium at or around the observed sub-surface damage. This hypothesis is addition- 
ally supported by the rough agreement of the depth scales at which sub-surface damage (Figure 4) and increased deuterium retention (Figure 7) occur.

\subsection{Deuterium desorption}

To gain access to information about detrapping energies of deuterium from background and evolving traps, TDS measurements were performed on the samples without layer system, from which also the depth profiles in Figure 5 originate. Figure 8 shows the total deuterium desorption flux carried by $\mathrm{HD}, \mathrm{D}_{2}, \mathrm{HDO}$ and $\mathrm{D}_{2} \mathrm{O}$ molecules. With respect to the heavy water contributions, the uncertainties mentioned in Section 2.4 must be kept in mind.

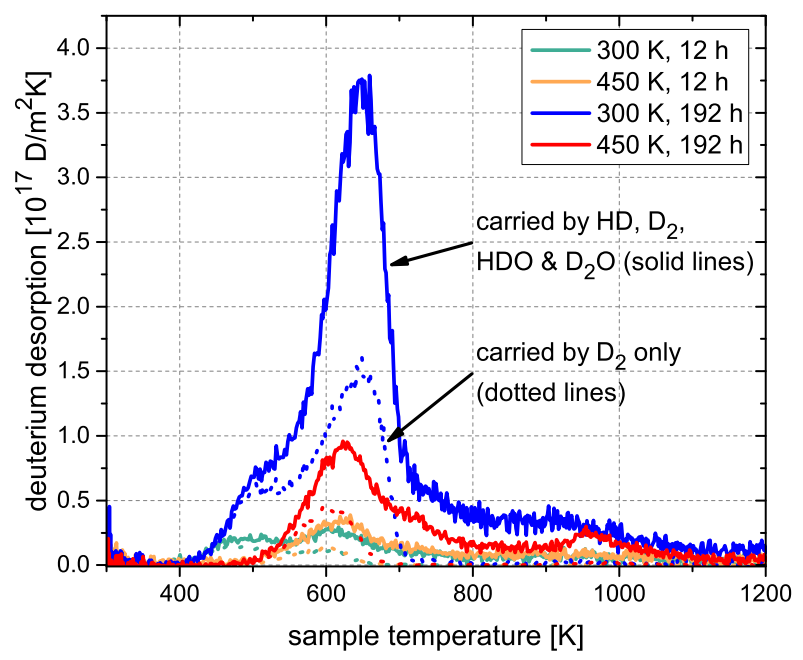

Figure 8: TDS analysis of retained deuterium in tungsten after deuterium-plasma exposure for $12 \mathrm{~h}$ and $192 \mathrm{~h}$ at $300 \mathrm{~K}$ and $450 \mathrm{~K}$. The data originates from the same samples as the depth profiles presented in Figure 5. Deuterium carried by HD, $\mathrm{D}_{2}, \mathrm{HDO}$ and $\mathrm{D}_{2} \mathrm{O}$ molecules was taken into account (solid lines). For these low desorption fluxes, the contribution carried by heavy water species is not negligible. The pure $\mathrm{D}_{2}$ contributions to the different spectra are also included (dotted lines). The fluxes are normalized to the plasma-exposed surface area.

Unfortunately, for the observed low desorption fluxes, the deuterium amount carried by heavy water species was comparable to the amount carried by $\mathrm{D}_{2}$, which is also displayed in Figure 8. The significant relative contribution of heavy water is suspected to be formed at the sample surface and maybe the chamber walls. It is thus a possible indicator for the presence of surface reactions during desorption that could affect the peak positions. The desorption fluxes carried by $\mathrm{D}_{2}$ have a peak around 600 to $650 \mathrm{~K}$. For the $300 \mathrm{~K}$ data a second peak appears around 460 to $500 \mathrm{~K}$. These peak positions are well within the range of peak positions reported in the literature for deuterium desorption from tungsten (compare, e.g., [5, 29, 30, 31, 32]).

In contrast to the observation of only a single desorption peak in the $\mathrm{D}_{2}$ signal from the samples exposed at $450 \mathrm{~K}$, the $300 \mathrm{~K}$ samples yield two desorption peaks. The second peak at a lower temperature may be caused by at least one additional trap type with a lower detrapping energy than the background traps active at $450 \mathrm{~K}$. It can also not be excluded that this second peak is caused or affected by different surface conditions present after plasma exposure at $300 \mathrm{~K}$. 


\subsection{Deuterium permeation}

To study the influence of the evolving sub-surface damage and the associated evolving trap concentration and deuterium retention on the permeation flux, the results of the microstructural analysis and retention measurements were combined with permeation measurement results. The permeated deuterium amount over deuterium-plasma exposure time for $300 \mathrm{~K}$ and $450 \mathrm{~K}$ exposure temperature, measured with the getter layer method described above, is displayed in Figure 9, where each data point represents one NRA measurement location. Data from typically five locations is displayed for each sample. Most of the $300 \mathrm{~K}$ data in Figure 9 was generated from measurements that were already used to produce the permeation data presented in [16], but were now evaluated with the improved evaluation procedure described in Section 2.3 .

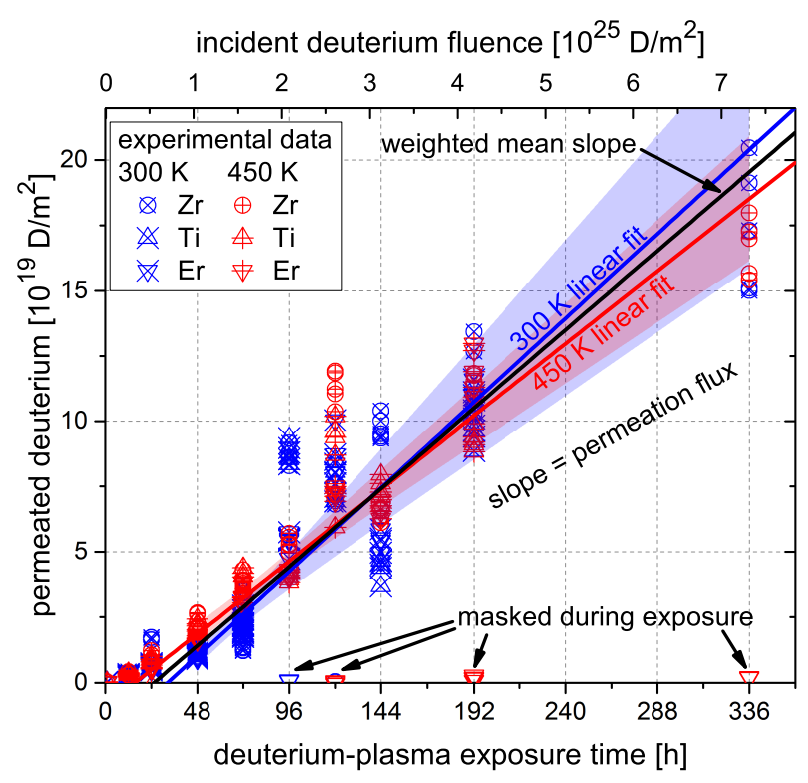

Figure 9: Permeated deuterium amount stored in the getter after deuterium-plasma exposure at $300 \mathrm{~K}$ and $450 \mathrm{~K}$ over exposure time. Linear fits with $95 \%$ confidence bands for both temperatures as well as a linear function with the weighted mean slope of both fit curves are also included. The slope is equal to the permeation flux. The fits were restricted to data points with exposure times of at least $48 \mathrm{~h}$ to take a certain lag-time into account. Data from samples that were exposed to the background deuterium gas, but masked from the incident ions during plasma exposure (open symbols without cross) has also been included to demonstrate the impermeability of the cover layer system. Most of the $300 \mathrm{~K}$ data originates from measurements that were already used to produce the permeation data published in [16].

Within the data scatter, the permeated deuterium amount increases roughly linearly with deuterium-plasma exposure time, as would be expected for a constant permeation flux. The data sets of each temperature have therefore been fitted with a linear function to determine the slope and thus the permeation flux. As a certain lag time in the occurrence of the permeation flux is expected, the linear fit regions were limited to data with exposure times of at least $48 \mathrm{~h}$. Due to the significant data scatter, also $95 \%$ confidence bands for the fits were included in the figure to give an impression of the associated uncertainties.

Taking the data scatter and the $95 \%$ confidence bands of the fits into account, the steady-state permeation flux at both temperatures is indistinguishable within the mea- 
surement uncertainties. By calculating the mean of the slopes from both fits, weighted with the number of samples used for each fit, a mean slope and thus mean steady-state permeation flux of $J_{\text {perm,exp }}=1.7 \times 10^{14} \mathrm{D} / \mathrm{m}^{2} \mathrm{~s}$ was determined. This is not identical with a linear fit to the data from both temperatures, as the lag times and thus the timeaxis intercepts are in general not expected to be identical even for an identical steady-state permeation flux. A linear curve with slope equal to $J_{\text {perm,exp }}$ is also included in Figure 9. The vertical-axis intercept has for simplicity been chosen as the weighted mean of the vertical-axis intercepts for both temperatures.

As already mentioned above, the upper-limit estimate for the rate with which deuterium gets trapped in traps that evolve in the NRA range below the plasma-exposed surface for an exposure temperature of $300 \mathrm{~K}$ is $R_{\mathrm{NRA}}^{\mathrm{D}}=1.3 \times 10^{14} \mathrm{D} / \mathrm{m}^{2} \mathrm{~s}$. This forms a loss channel for the solute deuterium, the concentration profile of which determines the permeation flux. Although this upper-limit estimate for the loss of solute deuterium to traps evolving below the plasma-exposed surface is of the same order of magnitude as the mean steady-state permeation flux $J_{\text {perm,exp }}=1.7 \times 10^{14} \mathrm{D} / \mathrm{m}^{2} \mathrm{~s}$, the presence of trap evolution at $300 \mathrm{~K}$ and its absence at $450 \mathrm{~K}$ exposure temperature cause no significant difference in the permeation flux at both temperatures. This effect will be elucidated based on the results of diffusion-trapping simulations in Section 4 .

\subsection{Maximum ratios of solute-deuterium to tungsten atoms}

A linear dependence of the solute-deuterium concentration and thus the ratio of solutedeuterium to tungsten atoms on the position between the implantation depth and the permeation side is predicted for the steady state of a constant permeation flux $J_{\text {perm }}$ that is unaffected by traps, e.g., in [33, 34, 35, 36]. Using this and Fick's first law

$$
J=-D \cdot \frac{\mathrm{d} c}{\mathrm{~d} x}
$$

with diffusion coefficient $D$, the maximum solute-deuterium concentration $c_{\text {max }}$ present in the sample below the plasma-exposed surface can be estimated by

$$
c_{\max }=\frac{J_{\mathrm{perm}} \cdot L}{D}
$$

(compare, e.g., [37]) if the sample thickness $L$ is much larger than the implantation depth $d_{\text {impl }}$ and a diffusion-limited boundary condition can be assumed at the permeation side.

Using the diffusion coefficient of hydrogen in tungsten reported by Frauenfelder [38], which is the most widely accepted literature value [4, 5], divided by $\sqrt{2}$ to take the isotope effect into account [10, 39], maximum ratios of solute-deuterium to tungsten atoms of about $8 \times 10^{-7}$ and $6 \times 10^{-9}$ were determined from the mean steady-state permeation flux $J_{\text {perm,exp }}$ for $300 \mathrm{~K}$ and $450 \mathrm{~K}$ exposure temperature, respectively. However, due to the large scatter of diffusion coefficients reported in the literature [5, 6], also the determined ratios of solute-deuterium to tungsten atoms have significant uncertainties.

\section{Modeling results}

To elucidate the experimental observation of an indistinguishable deuterium permeation flux measured for deuterium-plasma exposure at $300 \mathrm{~K}$ and $450 \mathrm{~K}$ despite significant damage and associated trap evolution only at the lower temperature, the experiments were 
modeled using the one-dimensional diffusion-trapping simulation code TESSIM [11, 39], again with Frauenfelder's diffusion coefficient [38] divided by $\sqrt{2}$ to take the isotope effect into account [10, 39]. The results of the diffusion-trapping simulations will be presented following a description of the chosen boundary conditions and the implantation distribution, which was implemented based on SDTrimSP [40] simulations.

\subsection{Boundary conditions}

The values for the surface recombination coefficients of hydrogen isotopes on tungsten reported in the literature have a very large data scatter [5, 41]. This impedes the choice of adequate boundary conditions for the simulations based on literature data. Due to the reasons discussed below, diffusion-limited boundary conditions implying

$$
c_{\text {solute }}(x=0, t)=c_{\text {solute }}(x=L, t)=0
$$

were used in all diffusion-trapping simulations presented in this report, as also suggested in [11, 39].

Regarding the permeation side during deuterium-plasma exposure, the cases with and without getter layer need to be distinguished. In the presence of a getter layer, recombination limitation can be excluded, as the deuterium atoms are dissolved in the getter atomically and thus do not need to recombine. Taking the data scatter into account, the permeated deuterium amounts measured with different getter materials are in reasonable agreement (compare Figure 9 and [16]). A possible interface barrier, which could lead to a deviation from the assumed boundary condition, therefore appears highly unlikely, because such a barrier would be expected to be different for different getter materials and thus disturb the agreement of the permeation data from samples with different getter materials [16]. In the absence of a getter layer, the depth profiles of Figure 5 show also no indications for recombination limitation, which would be expected to cause an accumulation of deuterium below the permeation side and thus result in a deviation from the negligible retention observed directly below the permeation-side surface.

Regarding the plasma-exposed side during deuterium-plasma exposure, the agreement of the permeation flux at $300 \mathrm{~K}$ and $450 \mathrm{~K}$ is an important indication for diffusion limitation. For recombination limitation at the plasma-exposed surface, in combination with the diffusion-limited condition at the permeation side motivated above, the temperature dependence of the recombination coefficient would affect the permeation flux [33, 34]. Unfortunately, values of the recombination coefficient reported in the literature have a large scatter and even differ with respect to the sign of its temperature dependence [5, 41]. It appears, however, improbable that the temperature dependence is by chance negligible. Thus, recombination limitation would be expected to disturb the observed agreement of the permeation flux at both exposure temperatures (compare [33, 34]). The steady-state permeation flux expected for different regimes of boundary conditions can be estimated based on the formulas given in 33. Assuming the above-mentioned values for the diffusion coefficient $D$, the incident deuteron flux density $J_{\text {incident }}$ and the sample thickness $L$ and assuming a reflection yield $Y_{\text {refl }}$ as well as an implantation depth based on the implantation simulations that will be presented in Section 4.2, the thus estimated steady-state permeation flux for diffusion limitation at both sides is of the same order of magnitude

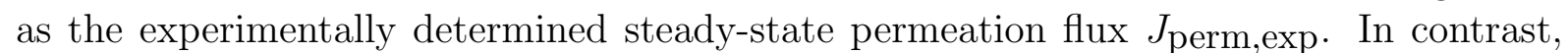
for recombination limitation at the plasma-exposed side and diffusion limitation at the 
permeation side, the thus estimated steady-state permeation flux assuming the abovementioned values and the combined surface recombination coefficient reported in [42] is about two to three orders of magnitude higher than $J_{\text {perm,exp. A higher recombination }}$ coefficient than reported in [42] would alleviate this discrepancy, however, it would also lead to a gradual transition to the case of diffusion limitation at both sides described above. Accordingly, a diffusion-limited boundary condition at the plasma-exposed side delivers the most reasonable description of our experimental observations in combination with the diffusion-limited boundary condition at the permeation side motivated above. Possibly, also particles incident from the plasma may assist in desorption by sputtering or abstraction.

For the TDS simulations, the situation at the surfaces may be more complex. Still, for simplicity, the diffusion-limited boundary conditions were also kept for the TDS simulations.

\subsection{Implantation profile}

An implantation source function to be used in the diffusion-trapping simulations was determined using the computer simulation code SDTrimSP [40] version 5.07 in static mode. The SDTrimSP implantation simulations were performed with $10^{6}$ projectiles reaching the sample under normal incidence. The energy distribution of the total incident differential deuteron flux density displayed in Figure 1 was used, modeling the incident deuterium molecules as individual incident deuterons.

The SDTrimSP input parameters surface binding energy, inelastic loss model, interaction potential and cutoff energy turned out to have a significant influence on the simulation result. Therefore, a parameter scan was performed and the resulting implantation profiles were used as input for diffusion-trapping simulations at $450 \mathrm{~K}$ with constant background traps only. This appears to be the best reference case since the constant background trap concentration has no effect on the steady-state permeation flux. The goal was to determine a set of parameters yielding a steady-state permeation flux that is in good agreement with the experimental result $J_{\text {perm,exp. Unfortunately, the surface binding }}$ energy is not well known and typically approximated by the heat of sublimation [43]. Within the framework of SDTrimSP, the surface binding energy does not only introduce an energy barrier for particles that attempt to leave the sample, but also accelerates incident particles [43]. While this is of minor importance at high incident energies, the low energies used in the present simulations are of the same order of magnitude as the standard value of the atomic surface binding energy for deuterium implemented in SDTrimSP [40] 5.07 of $1.1 \mathrm{eV}$. Optimizing for a good agreement of the resulting simulated steady-state permeation flux with the experimental result, a decrease of the surface binding energy, which had a strong impact on the permeation flux, down to $0 \mathrm{eV}$ turned out to be beneficial. The final implantation-model parameters used to generate a source function for the diffusion-trapping simulations presented in this report included a surface binding energy of $0 \mathrm{eV}$ as well as the $\mathrm{KrC}$ interaction potential and an equipartition of the LindhardScharff and Oen-Robinson inelastic loss models. This potential and inelastic loss model were also used, e.g., in [44]. Particles were followed to a cutoff energy that was chosen equal to the absolute value of Frauenfelder's activation energy for diffusion of $0.39 \mathrm{eV}$ [38] (compare, e.g., [4 for the value in eV). The implantation profile determined with SDTrimSP using this final parameter set is displayed in Figure 10, It has been fitted with a smooth function for implementation in the diffusion-trapping model. The simulated 
steady-state permeation flux resulting for this implantation profile and the corresponding reflection yield was less than ten percent higher than the experimental permeation flux

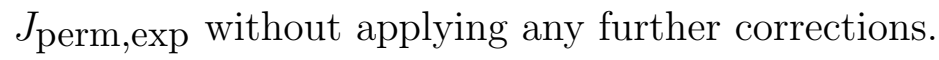

Since SDTrimSP uses the binary collision approximation (BCA), its validity in the present low-energy range needs to be discussed. It is known that the BCA loses its validity at very low energies. However, this effect deteriorates the results gradually and does not mean a sudden full loss of validity at a certain energy threshold [45. Due to the close match between experimental and simulated steady-state permeation flux using the SDTrimSP profile displayed in Figure 10 and the corresponding reflection yield, reasonable diffusion-trapping simulations based on this implantation-simulation result appear possible.

Still, as the influence of damage evolution on the permeation flux is the subject of the present study, it appeared reasonable to fully match the simulated steady-state permeation flux without trap evolution to the experimental steady-state permeation flux. Therefore, the reflection yield $Y_{\text {refl }}$ was increased slightly from $89 \%$, which resulted from the final SDTrimSP simulation, to $90 \%$ to perfectly match the simulated steady-state permeation flux at $450 \mathrm{~K}$ without trap evolution to the experimental steady-state permeation flux

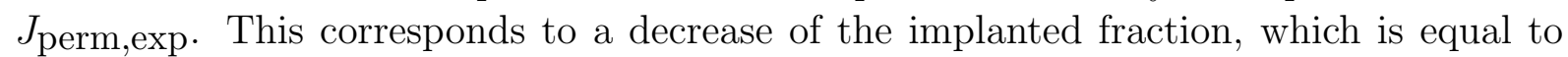
$\left(1-Y_{\text {refl }}\right)$, from $11 \%$ to $10 \%$.

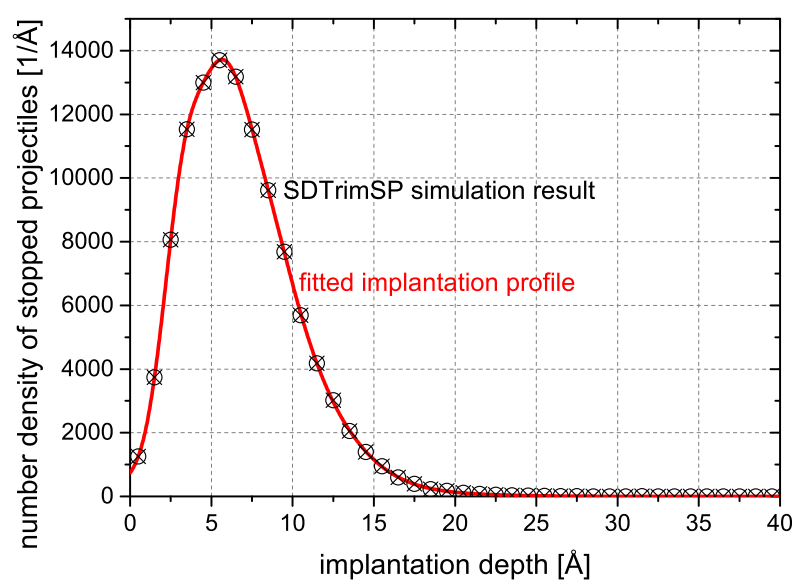

Figure 10: SDTrimSP implantation profile (circles with crosses) for the final parameter set, fitted with a smooth function (solid line).

This correction of the SDTrimSP result by modification of the reflection yield could have been achieved also by tuning the implantation depth or profile shape. In any case, since the length scales of sample size and evolving trap profile are orders of magnitude larger than the implantation depth, the exact shape of the profile is not crucial for the diffusion-trapping simulations as long as the same maximum solute concentration near the surface is reached.

\subsection{Influence of trap evolution on the permeation}

As discussed above, the trap density in the samples is assumed to be the sum of a background trap density represented by the number of background traps per tungsten atom $\rho_{0}^{\operatorname{tr}}$, which is constant in depth $x$ below the plasma-exposed surface and time $t$, and a trap density that evolves during the deuterium-plasma exposure and is represented by the number of evolving traps per tungsten atom $\rho_{\text {evolve }}^{\operatorname{tr}}(x, t)$. 
To elucidate the experimental results discussed above, three cases are most interesting for the diffusion-trapping simulations. The first two have no trap evolution during deuterium-plasma exposure at $450 \mathrm{~K}$ (case 1) and $300 \mathrm{~K}$ (case 2) corresponding to the experimental data from samples exposed at $450 \mathrm{~K}$ and the lower-limit estimate of trap evolution observed for samples exposed at $300 \mathrm{~K}$ (compare Figure 6), respectively. The third case with an evolving sub-surface trap profile at $300 \mathrm{~K}$ (case 3) represents the upper-limit estimate of the experimental data for $300 \mathrm{~K}$ exposure temperature.

\subsubsection{Without trap evolution}

In the first two simulation cases trap evolution occurs neither at $300 \mathrm{~K}$ (case 2) nor at $450 \mathrm{~K}$ (case 1). Thus, the total trap density is equal to the intrinsic background trap density and the total number of traps per tungsten atom is

$$
\rho_{\text {total }}^{\operatorname{tr}}(x, t)=\rho_{0}^{\operatorname{tr}}
$$

The resulting simulated permeated deuterium amount over time is compared with the experimental permeation data (which has already been presented in Figure 9) in Figure 11.

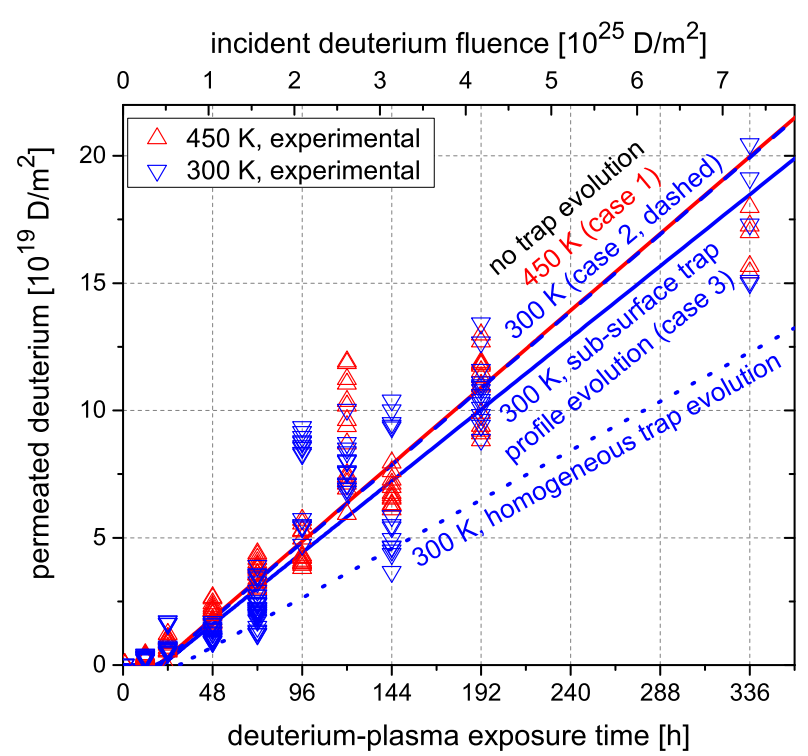

Figure 11: Simulated permeated deuterium amount over deuterium-plasma exposure time for different cases of trap evolution. In the first two cases without trap evolution at $300 \mathrm{~K}$ (case 2) and $450 \mathrm{~K}$ (case 1) the steady-state permeation flux is identical with nearly the same lag time. In the third case with trap evolution at $300 \mathrm{~K}$ with a profile peaked in the sub-surface region (case 3), the steady-state permeation flux is decreased only slightly compared to the cases without trap evolution. For comparison, an additional simulation with homogeneous trap evolution in the whole sample at $300 \mathrm{~K}$ has been included, where the decrease of the permeation flux is much more significant. This is the case although the total number of traps evolving per unit time and area is equal in both simulations with evolving traps. Experimental permeation data, which has already been shown in Figure 9, was added for comparison with the simulation results.

Within the experimental data scatter, the simulations for both exposure temperatures agree well with the experimental data. This is not surprising with respect to the slope, 
because the reflection yield has been adjusted to match the simulated steady-state permeation flux without trap evolution to the experimental value. However, in addition, the simulated lag times are in good agreement with experiment. The simulated ratios of solute-deuterium to tungsten atoms present in the sample during steady-state permeation after $192 \mathrm{~h}$ are displayed in Figure 12. As expected (compare Section 3.5, including [33, 34, 35, 36]), the ratios calculated with the diffusion-trapping model decrease linearly from a maximum below the plasma-exposed surface towards zero at the permeation side. Also, the values of the maximum solute-deuterium to tungsten atomic ratios are in excellent agreement with those determined using Equation 2 (compare Section 3.5).

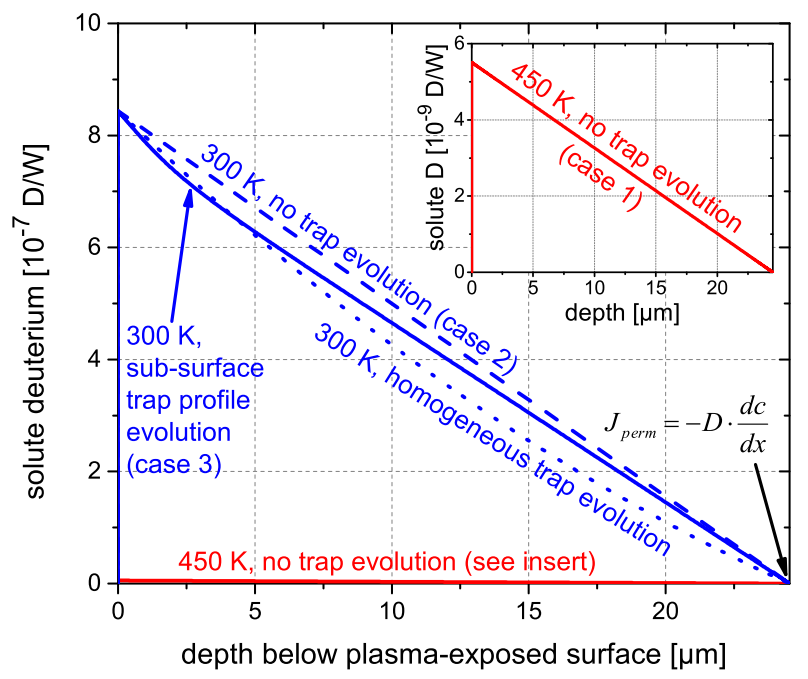

Figure 12: Simulated ratio of solute-deuterium to tungsten atoms in the sample during deuterium-plasma exposure after $192 \mathrm{~h}$ for constant background traps at $300 \mathrm{~K}$ (case 2) and $450 \mathrm{~K}$ (case 1), constant plus evolving sub-surface trap profile at $300 \mathrm{~K}$ (case 3) and constant plus homogeneously evolving traps at $300 \mathrm{~K}$. While the solute profiles decrease linearly from a maximum below the plasma-exposed surface to zero at the permeation side in the absence of evolving traps, the curves in presence of trap evolution deviate from a straight line. The slope at the permeation side is proportional to the permeation flux.

\subsubsection{Evolving sub-surface trap profile}

In the third simulation case of an evolving sub-surface trap profile at an exposure temperature of $300 \mathrm{~K}$ (case 3), the trap profile is not constant and can be described by the number of traps per tungsten atom

$$
\rho_{\text {total }}^{\operatorname{tr}}(x, t)=\rho_{0}^{\operatorname{tr}}+\sum_{i=1}^{N \text { evolve }} \rho_{\text {evolve }}^{\operatorname{tr}, i}(x, t),
$$

where $N_{\text {evolve }}^{\mathrm{tr}}$ is the number of types $i$ of evolving traps. The trap evolution has been modeled corresponding to the estimated upper limit for trap evolution in the NRA range below the plasma-exposed surface for $300 \mathrm{~K}$ exposure temperature mentioned above (see Figure 6).

For lack of a physical model that quantitatively describes damage and associated trap evolution due to the deuterium-plasma exposure, the shape of the evolving trap profile was estimated based on the measured deuterium depth profiles displayed in Figure 7. To 
exclude possible numerical artifacts due to steps resulting from the model used to evaluate the experimental data, the trap profile has been approximated with a continuously differentiable mathematical function that showed reasonable agreement with the measured depth profiles.

The equation

$$
\rho_{\text {evolve }}^{\operatorname{tr}, i}(x, t)=f_{i}(t) \cdot \frac{1}{2 \kappa} \cdot \exp \left(b_{1}(x)\right) \cdot \operatorname{erfc}\left(b_{2}(x)\right)
$$

with

$$
b_{1}(x)=\frac{1}{2} \cdot\left(\frac{\sigma}{\kappa}\right)^{2}-\frac{x-x_{0}}{\kappa}
$$

and

$$
b_{2}(x)=\frac{1}{\sqrt{2}} \cdot\left(\frac{\sigma}{\kappa}-\frac{x-x_{0}}{\sigma}\right),
$$

is based on an approximation of the exponentially modified Gaussian equation reported, e.g., in [46]. It has been chosen to model the evolving trap profile, as it resulted in the desired reasonable representation of the measured depth profiles as presented in Figure 7 . The underlying equation is not intended to provide a physical model for trap evolution, but has been introduced ad-hoc and chosen only due to its good agreement with the shape of the measured depth profiles. The model parameters $\sigma, \kappa$ and $x_{0}$ were determined by a least-squares fit to the $336 \mathrm{~h}$ depth profile in Figure 7, excluding the surface layer (see Section 3.2), and then kept constant for all exposure times. The time-dependent functions $f_{i}(t)$ in Equation 6 were, for simplicity, chosen as $f_{i}(t)=a_{\text {evolve }, i} \cdot t$. The $a_{\text {evolve }, i}$ were chosen such that the total model trap integral within the NRA range below the plasmaexposed side matched the estimated upper limit for evolving plus background traps in the same region, which has been mentioned in Section 3.2 and is included in Figure 6 .

In the most simple case of diffusion-limited boundary conditions at plasma-exposed and permeation side and a temperature-dependent but spatially constant diffusion coefficient, the steady-state permeation flux should be independent of temperature and solely be determined geometrically by implantation depth and sample thickness [33, 34]. However, this simple description is in general only applicable if no traps or only a constant trap distribution are present. While a constant trap distribution leads only to an increase of the time until the steady state is reached, a time-varying trap concentration can affect also the steady-state permeation flux. Still, the experimentally determined steady-state permeation flux was, within the experimental uncertainty, indistinguishable for deuterium-plasma exposure at $300 \mathrm{~K}$, where trap evolution was present, and at $450 \mathrm{~K}$, where no indications for trap evolution were found.

The diffusion-trapping simulation results with evolving sub-surface trap profile are also included in Figures 11 and 12. Figure 11 shows only a slight decrease of the simulated steady-state permeation flux due to the sub-surface trap profile evolution from $1.7 \times$ $10^{14} \mathrm{D} / \mathrm{m}^{2} \mathrm{~s}$ to $1.6 \times 10^{14} \mathrm{D} / \mathrm{m}^{2} \mathrm{~s}$, which still is well within the experimental data scatter. The simulation thus reproduces the indistinguishable steady-state permeation flux at $450 \mathrm{~K}$ without trap evolution and $300 \mathrm{~K}$ with sub-surface trap profile evolution, which was observed experimentally.

A lower ratio of solute-deuterium to tungsten atoms, mainly in the sub-surface region, compared to the situation without trap evolution at $300 \mathrm{~K}$, is visible in Figure 12. The only slightly different slope at the permeation side with sub-surface trap profile evolution 
at $300 \mathrm{~K}$ compared to the case without trap evolution at $300 \mathrm{~K}$ explains the only slight decrease in the steady-state permeation flux.

To reproduce the experimental TDS spectra of Figure 8 in the simulations, assuming one type of background traps and two types of evolving traps appeared reasonable. The detrapping energies of background traps $E_{\text {const }}^{\mathrm{TS}}$ and evolving traps $E_{\text {evolve }, i}^{\mathrm{TS}}$ as well as the relative contributions of the evolving trap types implemented in the model via the $a_{\text {evolve } i}$ were chosen by roughly matching the simulated TDS peak positions and relative heights to the measured deuterium desorption data displayed in Figure 8. The resulting simulated TDS spectra for $450 \mathrm{~K}$ without trap evolution (case 1) and $300 \mathrm{~K}$ with evolving sub-surface trap profile (case 3) are displayed in Figure 13. The experimentally observed single peak for $450 \mathrm{~K}$ exposure temperature and also the additional lower-temperature peak (or shoulder) for $300 \mathrm{~K}$ exposure temperature are well reproduced. The simulated peak positions agree well with experiment. For $300 \mathrm{~K}$ exposure temperature, deviations of the simulated peak heights from experiment result from the use of the upper-limit estimate for trap evolution (compare Figure 6), which causes a higher sub-surface retention than in the actual samples used for TDS. Furthermore, for both temperatures differences in the retention profiles, which will be discussed later, and heavy water effects, as discussed above, cause deviations in the peak heights of simulation and experiment.

As the frequency prefactor for detrapping is not well known, a typical literature value of $\nu_{\mathrm{TS}}=10^{13} \mathrm{~s}^{-1}$ [10, 39] was assumed for all trap types. Under this assumption, matching the simulated to the experimental peak position for desorption from a sample exposed for $192 \mathrm{~h}$ at $450 \mathrm{~K}$ yielded a detrapping energy of the background traps of about $\mathrm{E}_{\text {const }}^{\mathrm{TS}}=1.5 \mathrm{eV}$. As this peak is even higher for tungsten exposed at $300 \mathrm{~K}, E_{\text {const }}^{\mathrm{TS}}$ has also been assumed to be the main detrapping energy of the evolving traps and thus $\mathrm{E}_{\text {evolve }, 1}^{\mathrm{TS}}=\mathrm{E}_{\text {const }}^{\mathrm{TS}}$. To account for the second peak present at lower temperatures in the TDS measurements of the samples exposed at $300 \mathrm{~K}$, a part of the evolving traps was modeled with a detrapping energy of $\mathrm{E}_{\text {evolve }, 2}^{\mathrm{TS}}=1.25 \mathrm{eV}$. Both these detrapping energies are well within the range of literature values reported for detrapping energies of deuterium in tungsten [5]. However, the uncertainties associated with the TDS measurements discussed in Section 2.4 must be kept in mind and it should be mentioned that also desorption of hydrogen adsorbed on the tungsten surface can yield desorption peaks in this temperature range [47]. Therefore, the determined detrapping energies need to be taken with caution. The relative contributions of the evolving trap types, implemented via the $a_{\text {evolve }, i}$, were set to $a_{\text {evolve, } 1} / a_{\text {evolve }, 2}=2 / 1$. This ratio resulted in a lower-temperature peak after $12 \mathrm{~h}$ exposure at $300 \mathrm{~K}$ that was about as high as in experiment and a reasonable qualitative agreement of the peak structure shape after $192 \mathrm{~h}$. It is well possible that the real situation regarding the number of different trap types and their relative contributions is more complex than the presented model. But, as long as the deuterium retention profile evolution in the simulation is in agreement with the experimental data, the specific trap types and their relative contributions should be of minor importance for the simulation result regarding the permeation flux. This is the case for the present simulations, because the simulated filled fraction of both evolving trap types during steady-state permeation is nearly unity at $300 \mathrm{~K}$.

Simulated depth profiles of the deuterium trapped in the tungsten after plasma exposure without trap evolution at $450 \mathrm{~K}$ (case 1) and with sub-surface trap profile evolution at $300 \mathrm{~K}$ (case 3) are displayed in Figure 14. They are in reasonable agreement with the experimental profiles of Figure 5. The different height of the sub-surface retention peaks in experiment and simulation after exposure at $300 \mathrm{~K}$ results from the fact that 


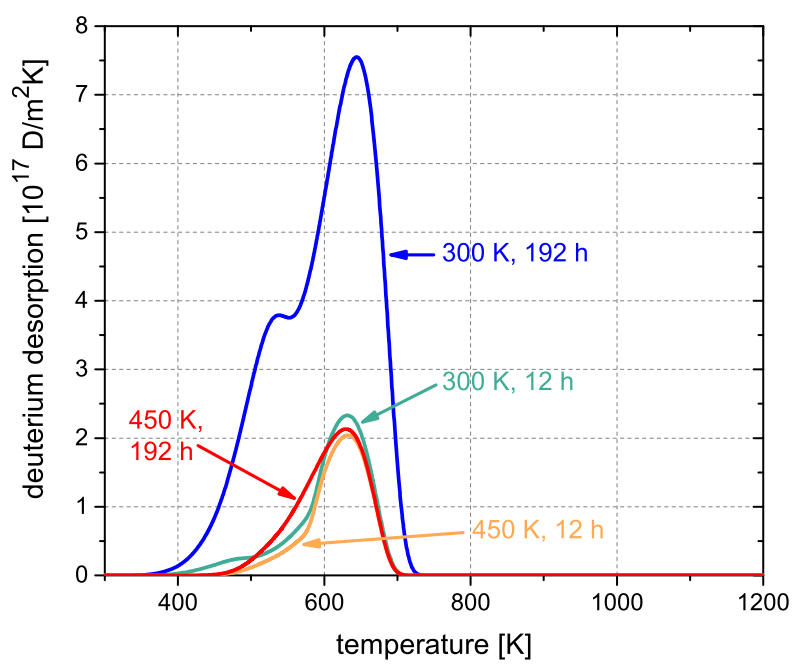

Figure 13: Simulated deuterium release during TDS for exposure without trap evolution at $450 \mathrm{~K}$ (case 1) and with evolving sub-surface trap profile at $300 \mathrm{~K}$ (case 3). The experimental observations displayed in Figure 8, which include a single peak for $450 \mathrm{~K}$ exposure temperature and an additional peak (or shoulder) at lower temperature for $300 \mathrm{~K}$ exposure temperature are well reproduced. Also the peak positions are in good agreement with the experimental TDS data. The peak heights deviate due to heavy water effects, such as sticking to the chamber walls, the model for $300 \mathrm{~K}$ exposure temperature using an upper-limit estimate for the trap evolution rate and deviations in the retained depth profiles.

the simulated retention is based on the upper-limit estimate for the traps per tungsten atom, which causes a retention that is higher than the actual retention measurement data without getter as displayed in Figure 6.

Comparing the experimental depth profiles in Figure 5 and the simulated ones in Figure 14, some deviations become apparent upon closer inspection. The background traps are filled up to a larger depth in the simulation after $12 \mathrm{~h}$. After $192 \mathrm{~h}$, the experimental depth profiles show a clear decrease of deuterium retention near the permeation side, which is not present in the simulation results. These deviations of the simulated from the experimental depth profiles indicate limitations of the used diffusion-trapping model. The amount of deuterium diffusing deep into the tungsten should not be different for experiment and simulation, because it is defined by the identical permeation flux in experiment and simulation without trap evolution. Therefore, the above-mentioned deviations may result from a less effective uptake of deuterium by the traps or a stronger detrapping in the experiment compared to the simulation. This effect would need to be stronger closer to the permeation side and similar at both temperatures. This might be caused, e.g., by a more complex energy landscape especially in the vicinity of traps, which may also depend on the local solute-deuterium concentration. The observed deviations could also be qualitatively explained by assuming that grain boundaries can be pathways for enhanced diffusion as suggested in [48]. Taking into account the dimensions of the grains in the material (compare Figure 3) and the sample thickness, it could be that close to the permeation side nearly all deuterium transport is concentrated in the grain boundaries. This would locally reduce bulk trapping while keeping permeation constant. 


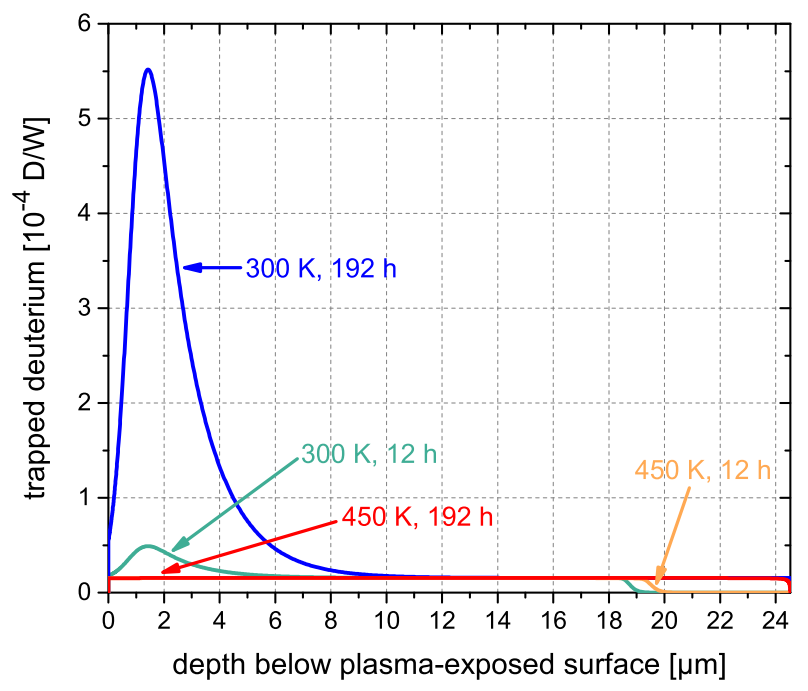

Figure 14: Simulated ratios of trapped deuterium to tungsten atoms after deuteriumplasma exposure. The overall structure of the experimental depth profiles in Figure 5 is well reproduced. Higher peaks after exposure at $300 \mathrm{~K}$ result from the use of the upperlimit estimate for trap evolution (compare Figure 6). Higher retention at larger depths in the simulation compared to experiment, e.g. below the permeation side after $192 \mathrm{~h}$ deuterium-plasma exposure (compare Figure 5), indicates limitations of the diffusiontrapping model (see text for a detailed discussion).

\subsubsection{Homogeneous trap evolution}

To elucidate the relation between the depth of trap evolution and its impact on the steady-state permeation flux, the evolution of a spatially homogeneous trap distribution was simulated in addition to the three experimentally motivated cases described above. To ensure comparability, the total number of traps evolving in the sample per unit time and area was chosen identical to the simulation including an evolving sub-surface trap profile at $300 \mathrm{~K}$ (case 3 ). The results of the simulation with homogeneous trap evolution at $300 \mathrm{~K}$ are also included in Figures 11 and 12 . Figure 11 demonstrates that homogeneous trap evolution leads to a stronger decrease of the steady-state permeation flux (compared to negligible trap evolution) than trap evolution close to the plasma-exposed surface. While the simulated steady-state permeation flux without trap evolution is $1.7 \times 10^{14} \mathrm{D} / \mathrm{m}^{2} \mathrm{~s}$, it is reduced to $1.1 \times 10^{14} \mathrm{D} / \mathrm{m}^{2} \mathrm{~s}$ with homogeneous trap evolution. As can be seen in Figure 12, also the ratio of solute-deuterium to tungsten atoms in the material is lower than in all the cases described previously, except in the sub-surface region, when compared to trap profile evolution peaked in this region, and directly below both surfaces. The difference in the slope at the permeation side, compared to the previous cases, also explains the lower permeation flux.

The stronger decrease of the steady-state permeation flux for homogeneously evolving traps compared to the evolving sub-surface trap profile can be interpreted in a random walk picture. Therein, solute deuterium atoms that become trapped in the vicinity of the plasma-exposed side would anyways have had a low probability to reach the permeation side. In contrast, solute deuterium atoms that become trapped close to the permeation side would have had a high probability to reach it, if they had not been trapped. Therefore, solute deuterium atoms that get trapped closer to the permeation side cause a stronger relative reduction of the steady-state permeation flux than those that get trapped further 
from the permeation side.

Figure 12, furthermore, demonstrates that in the presence of significant trap evolution, the determination of the maximum ratio of solute-deuterium to tungsten atoms based on the steady-state permeation flux using Equation 2 is no more reliable. This is because the solute-deuterium to tungsten atomic ratio and thus solute-deuterium concentration profile can strongly deviate from a straight line between a maximum below the plasma-exposed surface and the permeation side.

\section{$5 \quad$ Summary \& conclusions}

The influence of sub-surface damage evolution in tungsten during deuterium-plasma exposure on deuterium retention and permeation has been investigated. For the presented experimental conditions, sub-surface damage evolution was observed for plasma exposure at $300 \mathrm{~K}$, but not at $450 \mathrm{~K}$. The damage was visible in topview orientation-contrast SEM images and did not lead to detectable surface elevations, i.e. it was not visible in topographic-contrast SEM images of the surface. The presence of damage evolution at $300 \mathrm{~K}$ and its absence at $450 \mathrm{~K}$ exposure temperature are correlated with an evolving deuterium retention profile peaked in the sub-surface region that was also only observed for $300 \mathrm{~K}$ exposure temperature, but not for $450 \mathrm{~K}$. The correlation between sub-surface damage evolution and increased sub-surface deuterium retention strongly suggests the generation of additional traps for deuterium at or in the vicinity of the evolving subsurface damage. It is noteworthy that despite significant trap evolution only at $300 \mathrm{~K}$, an experimentally indistinguishable steady-state permeation flux was observed for both exposure temperatures.

The low incident ion energies (see Figure 1) and the associated shallow implantation profile exclude a direct kinetic generation of the sub-surface damage, which occurs even far beyond the implantation range. The presence of deuterium in the tungsten lattice is thus probably the most likely origin of the observed damage evolution. Estimates for the maximum ratio of solute-deuterium to tungsten atoms present during deuteriumplasma exposure of tungsten (a value that is generally difficult to access) in the presence and absence of damage evolution were determined based on the measured steady-state permeation flux. This is in contrast to many other studies, where the occurrence of material defects in tungsten during hydrogen-isotope ion implantation has been reported only in dependence on experimental parameters, such as the incident ion energy, flux and fluence, often also reporting the trapped deuterium amount after implantation (e.g., [49, 50, 51]). The maximum ratio of solute-deuterium to tungsten atoms in the tungsten during deuterium-plasma exposure at $300 \mathrm{~K}$, and thus in the presence of sub-surface damage evolution, was estimated to $8 \times 10^{-7}$, based on the measured permeation flux. In the case of negligible damage evolution during plasma exposure at $450 \mathrm{~K}$, the estimated maximum ratio of solute-deuterium to tungsten atoms was $6 \times 10^{-9}$. Regarding the uncertainty of the estimated ratios, the uncertainty in the diffusion coefficient is expected to be the dominant contribution. The solute-deuterium concentration is very likely crucial for the development of a microscopic theory of damage and associated trap evolution due to the presence of hydrogen isotopes in the tungsten lattice (compare, e.g., [4, 12, 13, 14]). Therefore, whenever possible, an inclusion of permeation flux measurements in experiments regarding the damage evolution in tungsten under hydrogen-isotope-plasma exposure is advantageous. Experimentally, the solute concentration can be assessed, e.g., by exposure of additional thin permeation samples, even if the sample of interest is too thick 
to exhibit a measurable permeation flux.

Various aspects and possible mechanisms relevant for sub-surface damage evolution and associated trap generation in tungsten due to the interaction with hydrogen-isotopes have been discussed in the literature based on experimental (e.g. [52, 53, 54]) as well as theoretical (e.g. [55, 56, 57]) investigations. However, a quantitative, physics-based model that fully describes this effect presently does not exist. Therefore, the evolving trap profile has been included in the presented diffusion-trapping model in an ad-hoc approach. The experimentally observed nearly identical permeation flux in the presence and absence of sub-surface damage and associated trap evolution at $300 \mathrm{~K}$ and $450 \mathrm{~K}$ exposure temperature, respectively, has been successfully reproduced in the diffusion-trapping simulations, which resulted in only a small decrease of the steady-state permeation flux due to a continuously increasing sub-surface trap profile. However, if the trap evolution is not limited to the sub-surface region, but occurs also deep in the material, it can lead to a much stronger decrease of the steady-state permeation flux, as has been demonstrated by modeling a homogeneous trap evolution with the same total number of traps evolving per unit time and area. It has also been shown that trap evolution can lead to a deviation of the simulated solute-deuterium to tungsten atomic ratio, and thus solute-deuterium concentration, during steady-state permeation from the most simple case of a linear decrease from a maximum below the plasma-exposed surface to zero at the permeation side. The simple equation used to estimate the maximum ratio of solute-deuterium to tungsten atoms based on the measured steady-state permeation flux (Equation 2) thus needs to be used with caution.

The damage and associated trap evolution in tungsten due to deuterium-plasma exposure are typically concentrated in the sub-surface region (compare, e.g., [9, 58]). This is not the case for defects and thus traps created by fusion neutrons, which extend deep into the material [4, 59]. If the total number of traps evolving in the tungsten per unit time and area due to hydrogen-isotope implantation and due to neutron damage would be comparable, then the impact of homogeneously distributed neutron damage on the steady-state permeation flux would be expected to be higher compared to the impact of near-surface trap generation by hydrogen isotopes, based on the presented simulations and within the assumptions made.

It has to be mentioned that Bauer et al. reported in [7] that a heavily blistered surface can reduce deep diffusion in tungsten under deuterium-plasma exposure. This may seem contradictory to the data presented in this report, which shows no significant influence of sub-surface damage evolution on the steady-state permeation flux. However, in contrast to the experiments described in the present report, the decreased deep diffusion described in 7 results from an increase of reemission due to the presence of ruptured blisters, and not from an evolving sub-surface trap concentration. It thus occurs in a different regime of much stronger sub-surface distortion.

Based on the results of the present report and the ones of Bauer et al. [7], three regimes of the effects of sub-surface damage evolution during deuterium-plasma exposure on deep diffusion in and steady-state permeation through tungsten can be distinguished. In this context, the assumptions made, e.g. with respect to the boundary conditions, have to be kept in mind. In the first regime of negligible damage evolution due to the interaction with deuterium, retention is dominated by the filling of the intrinsic background trap profile and the permeation flux saturates at a certain value. For diffusion-limited boundary conditions, this value is determined geometrically by implantation depth and sample thickness [33, 34]. In the second regime, sub-surface damage evolution leads to an evolu- 
tion of the sub-surface trap density. Thereby, sub-surface retention is increased, while the decrease of the steady-state permeation flux may be only small even for substantial trap evolution. Finally, the third regime of massive blistering, described in [7], shows a decrease of deep diffusion and thus steady-state permeation. However, sub-surface retention appears likely to be increased compared to the first regime, even if most blisters may be ruptured. This is because an increased retention could result from trapping at defects created in the vicinity of the blisters such as those reported in [60]. When hydrogen-isotope retention and steady-state permeation are intended to be minimized, the first regime is preferable to the second one. With respect to a fusion reactor, where also a possible contamination of the plasma with tungsten needs to be taken into account, the ranking of the first versus the third regime depends on the retention increase and severity of the degradation of the structural integrity of the tungsten surface due to massive blistering. Furthermore, additional effects due to admixture of helium and seeded impurities as well as defect creation by neutrons need to be taken into account.

\section{Acknowledgments}

The authors would like to thank Joachim Dorner, Michael Fußeder and Stefan Schindler for support regarding the ion-beam analysis, Thomas Dürbeck regarding the TDS measurements, Gabriele Matern regarding the microstructural analysis and Till Höschen regarding the XPS measurements, all from the Max-Planck-Institut für Plasmaphysik (IPP). Furthermore, valuable discussions with Dr. Wolfgang Jacob (IPP), advice from Dr. Andreas Mutzke (IPP) regarding SDTrimSP and improvements of MultiSIMNRA implemented by Dr. Cleber Rodrigues from the Instituto de Física da Universidade São Paulo are acknowledged. S. Kapser is grateful for support by the International Helmholtz Graduate School for Plasma Physics (HEPP), which is associated with the TUM Graduate School.

\section{References}

[1] Y. Ueda, J.W. Coenen, G. De Temmerman, R.P. Doerner, J. Linke, V. Philipps, and E. Tsitrone. Research status and issues of tungsten plasma facing materials for ITER and beyond. Fusion Engineering and Design, 89(7):901 - 906, 2014. ISSN 09203796. doi:10.1016/j.fusengdes.2014.02.078. Proceedings of the 11th International Symposium on Fusion Nuclear Technology-11 (ISFNT-11) Barcelona, Spain, 15-20 September, 2013.

[2] J. Knaster, A. Moeslang, and T. Muroga. Materials research for fusion. Nature Physics, 12:424-434, 2016. doi:10.1038/nphys3735.

[3] J. Ongena, R. Koch, R. Wolf, and H. Zohm. Magnetic-confinement fusion. Nature Physics, 12:398-410, 2016. doi:10.1038/nphys3745.

[4] Joachim Roth and Klaus Schmid. Hydrogen in tungsten as plasma-facing material. Physica Scripta, T145:014031, 2011. doi:10.1088/0031-8949/2011/T145/014031.

[5] Rion A Causey. Hydrogen isotope retention and recycling in fusion reactor plasmafacing components. Journal of Nuclear Materials, 300(2):91 - 117, 2002. ISSN 0022-3115. doi:10.1016/S0022-3115(01)00732-2. 
[6] T Tanabe. Review of hydrogen retention in tungsten. Physica Scripta, T159:014044, 2014. doi:10.1088/0031-8949/2014/T159/014044.

[7] J. Bauer, T. Schwarz-Selinger, K. Schmid, M. Balden, A. Manhard, and U. von Toussaint. Influence of near-surface blisters on deuterium transport in tungsten. Nuclear Fusion, 57(8):086015, 2017. doi:10.1088/1741-4326/aa7212.

[8] Armin Manhard, Martin Balden, and Udo von Toussaint. Blister formation on rough and technical tungsten surfaces exposed to deuterium plasma. Nuclear Fusion, 57 (12):126012, 2017. doi:10.1088/1741-4326/aa82c8.

[9] Y. Zayachuk, A. Manhard, M.H.J. 't Hoen, W. Jacob, P.A. Zeijlmans van Emmichoven, and G. van Oost. Depth profiling of the modification induced by high-flux deuterium plasma in tungsten and tungsten-tantalum alloys. Nuclear Fusion, 54(12): 123013, 2014. doi:10.1088/0029-5515/54/12/123013.

[10] K. Schmid, U. von Toussaint, and T. Schwarz-Selinger. Transport of hydrogen in metals with occupancy dependent trap energies. Journal of Applied Physics, 116 (13):134901, 2014. doi:10.1063/1.4896580.

[11] K Schmid. Diffusion-trapping modelling of hydrogen recycling in tungsten under ELM-like heat loads. Physica Scripta, T167:014025, 2016. doi:10.1088/00318949/T167/1/014025.

[12] V Kh Alimov, W M Shu, J Roth, K Sugiyama, S Lindig, M Balden, K Isobe, and T Yamanishi. Surface morphology and deuterium retention in tungsten exposed to low-energy, high flux pure and helium-seeded deuterium plasmas. Physica Scripta, T138:014048, 2009. doi:10.1088/0031-8949/2009/T138/014048.

[13] S. Markelj, T. Schwarz-Selinger, A. Založnik, M. Kelemen, P. Vavpetič, P. Pelicon, E. Hodille, and C. Grisolia. Deuterium retention in tungsten simultaneously damaged by high energy W ions and loaded by D atoms. Nuclear Materials and Energy, 12: 169-174, 2016. ISSN 2352-1791. doi:10.1016/j.nme.2016.11.010. Proceedings of the 22nd International Conference on Plasma Surface Interactions 2016, 22nd PSI.

[14] K Schmid, J Bauer, T Schwarz-Selinger, S Markelj, U v Toussaint, A Manhard, and $\mathrm{W}$ Jacob. Recent progress in the understanding of $\mathrm{H}$ transport and trapping in $\mathrm{W}$. Physica Scripta, T170:014037, 2017. doi:10.1088/1402-4896/aa8de0.

[15] A Manhard, T Schwarz-Selinger, and W Jacob. Quantification of the deuterium ion fluxes from a plasma source. Plasma Sources Science and Technology, 20(1):015010, 2011. doi:10.1088/0963-0252/20/1/015010.

[16] Stefan Kapser, Armin Manhard, and Udo von Toussaint. Measuring deuterium permeation through tungsten near room temperature under plasma loading using a getter layer and ion-beam based detection. Nuclear Materials and Energy, 12:703708, 2017. ISSN 2352-1791. doi:10.1016/j.nme.2016.11.019. Proceedings of the 22nd International Conference on Plasma Surface Interactions 2016, 22nd PSI.

[17] L. Gao, W. Jacob, T. Schwarz-Selinger, and A. Manhard. Deuterium implantation into tungsten nitride: Negligible diffusion at 300 K. Journal of Nuclear Materials, 451(1-3):352-355, 2014. doi:10.1016/j.jnucmat.2014.04.029. 
[18] M. Mayer, E. Gauthier, K. Sugiyama, and U. von Toussaint. Quantitative depth profiling of deuterium up to very large depths. Nuclear Instruments and Methods in Physics Research Section B: Beam Interactions with Materials and Atoms, 267(3): 506 - 512, 2009. ISSN 0168-583X. doi:10.1016/j.nimb.2008.11.033.

[19] B. Wielunska, M. Mayer, T. Schwarz-Selinger, U. von Toussaint, and J. Bauer. Cross section data for the $\mathrm{D}\left({ }^{3} \mathrm{He}, \mathrm{p}\right){ }^{4} \mathrm{He}$ nuclear reaction from 0.25 to $6 \mathrm{MeV}$. Nuclear Instruments and Methods in Physics Research Section B: Beam Interactions with Materials and Atoms, 371:41 - 45, 2016. ISSN 0168-583X. doi:10.1016/j.nimb.2015.09.049. The 22nd International Conference on Ion Beam Analysis (IBA 2015).

[20] K. Schmid and U. von Toussaint. Statistically sound evaluation of trace element depth profiles by ion beam analysis. Nuclear Instruments and Methods in Physics Research Section B: Beam Interactions with Materials and Atoms, 281:64 - 71, 2012. ISSN 0168-583X. doi:10.1016/j.nimb.2012.03.024.

[21] M. Mayer. SIMNRA User's Guide. Max-Planck-Institut für Plasmaphysik, Garching, Germany, April 1997. URL http://hdl.handle.net/11858/ 00-001M-0000-0027-6157-F. Report IPP 9/113.

[22] M. Mayer. Improved physics in SIMNRA 7. Nuclear Instruments and Methods in Physics Research Section B: Beam Interactions with Materials and Atoms, 332:176 - 180, 2014. ISSN 0168-583X. doi:10.1016/j.nimb.2014.02.056. 21st International Conference on Ion Beam Analysis.

[23] T. F. Silva, C.L. Rodrigues, M. Mayer, M.V. Moro, G.F. Trindade, F.R. Aguirre, N. Added, M.A. Rizzutto, and M.H. Tabacniks. MultiSIMNRA: A computational tool for self-consistent ion beam analysis using SIMNRA. Nuclear Instruments and Methods in Physics Research Section B: Beam Interactions with Materials and Atoms, 371:86 - 89, 2016. ISSN 0168-583X. doi:10.1016/j.nimb.2015.10.038. The 22nd International Conference on Ion Beam Analysis (IBA 2015).

[24] E. Salançon, T. Dürbeck, T. Schwarz-Selinger, F. Genoese, and W. Jacob. Redeposition of amorphous hydrogenated carbon films during thermal decomposition. Journal of Nuclear Materials, 376(2):160 - 168, 2008. ISSN 0022-3115. doi:10.1016/j.jnucmat.2008.02.070

[25] P. Wang, W. Jacob, L. Gao, T. Dürbeck, and T. Schwarz-Selinger. Comparing deuterium retention in tungsten films measured by temperature programmed desorption and nuclear reaction analysis. Nuclear Instruments and Methods in Physics Research Section B: Beam Interactions with Materials and Atoms, 300(Supplement C):54 61, 2013. ISSN 0168-583X. doi:10.1016/j.nimb.2013.01.057.

[26] Relative Sensitivity. HIDEN ANALYTICAL LTD, 420 Europa Boulevard, Warrington, WA5 7UN, England. URL https://www.hidenanalytical.de/wp-content/ uploads/pdf/RS_Measurement_of_Gases_-_Hiden_Analytical_App_Note_282. pdf. Gas Analysis Application Note 282 (accessed April 13, 2017).

[27] A. Manhard, K. Schmid, M. Balden, and W. Jacob. Influence of the microstructure on the deuterium retention in tungsten. Journal of Nuclear Materials, 415(1, Supplement):S632 - S635, 2011. ISSN 0022-3115. doi:10.1016/j.jnucmat.2010.10.045. 
Proceedings of the 19th International Conference on Plasma-Surface Interactions in Controlled Fusion.

[28] K. Heinola, T. Ahlgren, K. Nordlund, and J. Keinonen. Hydrogen interaction with point defects in tungsten. Physical Review B, 82(9), 2010. doi: 10.1103/physrevb.82.094102.

[29] O V Ogorodnikova, T Schwarz-Selinger, K Sugiyama, T Dürbeck, and W Jacob. Deuterium retention in different tungsten grades. Physica Scripta, T138:014053, 2009. doi:10.1088/0031-8949/2009/T138/014053.

[30] A Manhard, U v Toussaint, T Dürbeck, K Schmid, and W Jacob. Statistical analysis of blister bursts during temperature-programmed desorption of deuterium-implanted polycrystalline tungsten. Physica Scripta, T145:014038, 2011. doi:10.1088/0031$8949 / 2011 / \mathrm{T} 145 / 014038$

[31] O. V. Ogorodnikova, T. Schwarz-Selinger, K. Sugiyama, and V. Kh. Alimov. Deuterium retention in tungsten exposed to low-energy pure and heliumseeded deuterium plasmas. Journal of Applied Physics, 109(1):013309, 2011. doi:10.1063/1.3505754.

[32] S. Ryabtsev, Yu. Gasparyan, M. Zibrov, A. Shubina, and A. Pisarev. Deuterium thermal desorption from vacancy clusters in tungsten. Nuclear Instruments and Methods in Physics Research Section B: Beam Interactions with Materials and Atoms, 382 (Supplement C):101 - 104, 2016. ISSN 0168-583X. doi:10.1016/j.nimb.2016.04.038. The 21st International workshop on Inelastic Ion Surface Collisions (IISC-21).

[33] W. M. Shu, K. Okuno, and Y. Hayashi. General model for ion-driven permeation at steady state: new transport parameters. The Journal of Physical Chemistry, 97(17): 4497-4499, 1993. doi:10.1021/j100119a040.

[34] T. Tanabe, Y. Furuyama, and S. Imoto. Hydrogen ion driven permeation through metals. Journal of Nuclear Materials, 145-147:305-308, 1987. doi:10.1016/00223115(87)90349-7.

[35] B.L. Doyle. A simple theory for maximum $\mathrm{H}$ inventory and release: A new transport parameter. Journal of Nuclear Materials, 111-112:628-635, 1982. doi:10.1016/00223115(82)90277-x.

[36] O.K. Brice and B.L. Doyle. Steady state hydrogen transport in solids exposed to fusion reactor plasmas. Journal of Nuclear Materials, 120(2-3):230-244, 1984. doi:10.1016/0022-3115(84)90061-8.

[37] Ikuji Takagi, Kouta Kodama, Kazuo Shin, Kunio Higashi, Hideki Zushi, Tohru Mizuuchi, Tohru Senjyu, Masahiro Wakatani, and Tokuhiro Obiki. Deuterium plasmadriven permeation in heliotron $\mathrm{E}$ during discharge cleaning and in a small plasma device. Fusion Technology, 25(2):137-146, 1994. doi:10.13182/fst94-a30263.

[38] R. Frauenfelder. Solution and diffusion of hydrogen in tungsten. Journal of Vacuum Science and Technology, 6(3):388-397, 1969. doi:10.1116/1.1492699. 
[39] K. Schmid, V. Rieger, and A. Manhard. Comparison of hydrogen retention in W and W/Ta alloys. Journal of Nuclear Materials, 426(1):247 - 253, 2012. ISSN 0022-3115. doi:10.1016/j.jnucmat.2012.04.003.

[40] A. Mutzke, R. Schneider, W. Eckstein, and R. Dohmen. SDTrimSP Version 5.00. IPP Report 12/8, Max-Planck-Institut für Plasmaphysik, 85748 Garching, Germany, April 2011. URL http://hdl.handle.net/11858/00-001M-0000-0026-EAF9-A.

[41] H.T. Lee, H. Tanaka, Y. Ohtsuka, and Y. Ueda. Ion-driven permeation of deuterium through tungsten under simultaneous helium and deuterium irradiation. Journal of Nuclear Materials, 415(1):S696-S700, 2011. doi:10.1016/j.jnucmat.2010.12.023.

[42] R. A. Anderl, D. F. Holland, G. R. Longhurst, R. J. Pawelko, C. L. Trybus, and C. H. Sellers. Deuterium transport and trapping in polycrystalline tungsten. Fusion Technology, 21(2P2):745-752, 1992. doi:10.13182/fst92-a29837.

[43] Wolfgang Eckstein. Computer Simulation of Ion-Solid Interactions, chapter Thermal Vibrations and Specific Energies, pages 73-82. Springer Berlin Heidelberg, 1991. ISBN 978-3-642-73513-4. doi:10.1007/978-3-642-73513-4_6.

[44] Wolfgang Eckstein. Sputtering, Reflection and Range Values for Plasma Edge Codes. Max-Planck-Institut für Plasmaphysik, Garching, Germany, 1998. URL http:// hdl.handle.net/11858/00-001M-0000-0027-6091-4. IPP Report 9/117.

[45] Wolfgang Eckstein and Herbert M. Urbassek. Sputtering by Particle Bombardment: Experiments and Computer Calculations from Threshold to MeV Energies, chapter Computer Simulation of the Sputtering Process, pages 21-31. Springer Berlin Heidelberg, 2007. ISBN 978-3-540-44502-9. doi:10.1007/978-3-540-44502-9_2.

[46] Douglas Hanggi and Peter W. Carr. Errors in exponentially modified gaussian equations in the literature. Analytical Chemistry, 57(12):2394-2395, 1985. doi:10.1021/ac00289a051.

[47] P. W. Tamm and L. D. Schmidt. Binding states of hydrogen on tungsten. The Journal of Chemical Physics, 54(11):4775-4787, 1971. doi:10.1063/1.1674753.

[48] U von Toussaint, S Gori, A Manhard, T Höschen, and C Höschen. Molecular dynamics study of grain boundary diffusion of hydrogen in tungsten. Physica Scripta, T145:014036, 2011. doi:10.1088/0031-8949/2011/t145/014036.

[49] C.N. Taylor, M. Shimada, and B.J. Merrill. Deuterium retention and blistering in tungsten foils. Nuclear Materials and Energy, 12:689-693, 2017. ISSN 2352-1791. doi:10.1016/j.nme.2016.12.004. Proceedings of the 22nd International Conference on Plasma Surface Interactions 2016, 22nd PSI.

[50] G.-N. Luo, W.M. Shu, and M. Nishi. Incident energy dependence of blistering at tungsten irradiated by low energy high flux deuterium plasma beams. Journal of Nuclear Materials, 347(1):111 - 117, 2005. ISSN 0022-3115. doi:10.1016/j.jnucmat.2005.07.014.

[51] M.H.J. 't Hoen, M. Balden, A. Manhard, M. Mayer, S. Elgeti, A.W. Kleyn, and P.A. Zeijlmans van Emmichoven. Surface morphology and deuterium retention of 
tungsten after low- and high-flux deuterium plasma exposure. Nuclear Fusion, 54(8): 083014, 2014. doi:10.1088/0029-5515/54/8/083014.

[52] S Lindig, M Balden, V Kh Alimov, T Yamanishi, W M Shu, and J Roth. Subsurface morphology changes due to deuterium bombardment of tungsten. Physica Scripta, T138:014040, 2009. doi:10.1088/0031-8949/2009/t138/014040.

[53] V.Kh. Alimov, W.M. Shu, J. Roth, S. Lindig, M. Balden, K. Isobe, and T. Yamanishi. Temperature dependence of surface topography and deuterium retention in tungsten exposed to low-energy, high-flux D plasma. Journal of Nuclear Materials, 417(1-3): 572-575, 2011. doi:10.1016/j.jnucmat.2011.01.088.

[54] H.Y. Xu, W. Liu, G.N. Luo, Y. Yuan, Y.Z. Jia, B.Q. Fu, and G. De Temmerman. Blistering on tungsten surface exposed to high flux deuterium plasma. Journal of Nuclear Materials, 471:51-58, 2016. doi:10.1016/j.jnucmat.2015.12.025.

[55] S.C. Middleburgh, R.E. Voskoboinikov, M.C. Guenette, and D.P. Riley. Hydrogen induced vacancy formation in tungsten. Journal of Nuclear Materials, 448(1-3):270275, 2014. doi:10.1016/j.jnucmat.2014.02.014.

[56] Donald F. Johnson and Emily A. Carter. Hydrogen in tungsten: Absorption, diffusion, vacancy trapping, and decohesion. Journal of Materials Research, 25(02): 315-327, 2010. doi:10.1557/jmr.2010.0036.

[57] Daiji Kato, Hirotomo Iwakiri, and Kazunori Morishita. Formation of vacancy clusters in tungsten crystals under hydrogen-rich condition. Journal of Nuclear Materials, 417 (1-3):1115-1118, 2011. doi:10.1016/j.jnucmat.2010.12.211.

[58] V. Kh. Alimov, J. Roth, and M. Mayer. Depth distribution of deuterium in singleand polycrystalline tungsten up to depths of several micrometers. Journal of Nuclear Materials, 337-339:619-623, 2005. doi:10.1016/j.jnucmat.2004.10.082.

[59] W.R. Wampler and R.P. Doerner. The influence of displacement damage on deuterium retention in tungsten exposed to plasma. Nuclear Fusion, 49(11):115023, 2009. doi:10.1088/0029-5515/49/11/115023.

[60] A. Manhard, U. von Toussaint, M. Balden, S. Elgeti, T. Schwarz-Selinger, L. Gao, S. Kapser, T. Płociński, J. Grzonka, M. Gloc, and Ł. Ciupiński. Microstructure and defect analysis in the vicinity of blisters in polycrystalline tungsten. Nuclear Materials and Energy, 12:714-719, 2017. doi:10.1016/j.nme.2016.10.014. Proceedings of the 22nd International Conference on Plasma Surface Interactions 2016, 22nd PSI. 